\title{
In Situ Observation of Metal to Metal Oxide Progression: A Study of Charge Transfer Phenomenon at Ru-CuO Interface
}

Alexander Porkovich, ${ }^{*},{ }^{\dagger}$ Zakaria Ziadi, ${ }^{\dagger}$ Pawan Kumar, ${ }^{\dagger}$ Joseph Kioseoglou, ${ }^{\ddagger}$ Nan Jian, ${ }^{\dagger}, \S$ Lin Weng, ${ }^{\dagger}$ Stephan Steinhauer, ${ }^{\dagger}$ Jerome Vernieres, ${ }^{\dagger}, \perp$ Panagiotis Grammatikopoulos ${ }^{\dagger}$ and Mukhles Sowwan $*, \dagger$

†Nanoparticles by Design Unit, Okinawa Institute of Science and Technology (OIST) Graduate University, 1919-1 Tancha, OnnaSon, Okinawa 904-0495, Japan

$\$$ Department of Physics, Aristotle University of Thessaloniki, GR-54124 Thessaloniki, Greece

$\S$ Institute of Nanosurface Science and Engineering, Shenzhen University, Nanhai Ave. 3688, Shenzhen, Guangdong, China

${ }^{\perp}$ College of Engineering, Nanomaterials Lab, Swansea University, Bay Campus, Fabian way, SA1 8EN Swansea, UK

Section S1. Device Fabrication:

Figure S1. Schematic of Lithographic Process

Figure S2. Scanning electron microscope (SEM) images of the device

Section S2. Conductometric measurements of individual devices with conditions:

Figure S3. Current through undecorated and decorated devices at $200^{\circ} \mathrm{C}$ and a $1000 \mathrm{sccm}$ airflow

Figure S4. Current through undecorated and decorated devices at $250^{\circ} \mathrm{C}$ and a $1000 \mathrm{sccm}$ airflow

Section S3. High resolution X-ray photoelectron spectroscopy (XPS):

Figure $\mathrm{S} 5 . \mathrm{Cu} 2 \mathrm{p}$ XPS spectra of undecorated $\mathrm{CuO}$, as-desposited $\mathrm{Ru}-\mathrm{CuO}$ and annealed (for 1 and 5 hours) $\mathrm{Ru}-\mathrm{CuO}$

Figure $\mathrm{S} 6 . \mathrm{O}$ 1s XPS spectra of undecorated $\mathrm{CuO}$, as-desposited $\mathrm{Ru}-\mathrm{CuO}$ and annealed (for 1 and 5 hours) $\mathrm{Ru}-\mathrm{CuO}$

Section S4. (Scanning) Transmission electron microscopy ((S)TEM) analysis of Ru nanoclusters (NCs) and $\mathrm{Ru} \mathrm{NCs}$ on $\mathrm{CuO}$ nanowires (NWs):

Figure S7. Size and shape distribution of Ru NCs

Figure S8. TEM images of different size single crystal Ru NCs on amorphous carbon grid

Table S1. $d$-spacings of Ru NCs from Figure S8

Figure S9. STEM high angle annular dark field (HAADF) images of single crystal Ru NCs on amorphous carbon grid

Table S2. $d$-spacings of Ru NCs from Figure S9 
Table S3. $d$-spacings of Ru NCs on $\mathrm{CuO}$ NWs

Figure S10. Full images of Figure 3

Figure S11. Filtered image of icoshedral image on $\mathrm{CuO}$ NW

Section S5. STEM observation of nanocluster-nanowire system during transient periods:

Figure S12. Image and analysis of Ru NC after 45 minutes of ex-situ annealing in dry air.

Figure S13. Image and analysis of Ru NC after 66 minutes of ex-situ annealing in dry air.

Figure S14. Image of monoclinic $\mathrm{RuO}_{4} \mathrm{NC}$ observed during transient periods

Figure S15. Image and analysis of fully oxidised $\mathrm{RuO}_{2} @ \mathrm{Ru} \mathrm{NC}$

Section S6. Atomistic Models of Ru NC-CuO surface:

Figure $\mathrm{S} 16$. Relaxed model reconstruction of $\mathrm{Ru} \mathrm{NC}$ on $\mathrm{CuO} \mathrm{NW}$ from figure 3, viewed along surface. Subsequently, charge profiles of $\mathrm{Ru} \mathrm{NC}, \mathrm{CuO}$ NW surface and Ru NC on $\mathrm{CuO}$ surface are also displayed.

Figure S17. The profiles from figure S16 viewed along the perpendicular direction.

Figure S18. Relaxed model reconstruction of a hcp Ru NC on CuO NW with facets (1011) on $\mathrm{CuO}$ (111) surface, viewed along [1120]. Subsequently, charge profiles of $\mathrm{Ru} \mathrm{NC}, \mathrm{CuO}$ NW surface and $\mathrm{Ru} \mathrm{NC}$ on $\mathrm{CuO}$ surface are also displayed.

Figure S19. The profiles from figure S18 viewed along the perpendicular direction. Section S7. PeakForce Kelvin probe force microscopy (PF-KPFM) supplement and CuO NW analysis:

Figure S20. Schematic of the PF-KPFM set-up

Figure S21. PF-KPFM image and analysis of pristine $\mathrm{CuO}$ NW

Figure S22. Band diagrams before and after contact between nanoclusters and nanowires Section S8. Acetone gas sensing measurments:

Figure S23. Gas sensing response of $\mathrm{CuO}$ NWs to acetone at different temperatures Figure S24. Gas sensing response of Ru NCs on $\mathrm{CuO}$ NWs to acetone at $200^{\circ} \mathrm{C}$.

Section S9. Band structure of $\mathrm{CuO}$ atomistic model:

Figure $\mathrm{S} 25 . \mathrm{CuO} \mathrm{NW}$ bandstructure as calculated through DFT Section S10. Supplementary information references 


\section{S1. Device Fabrication}

a)

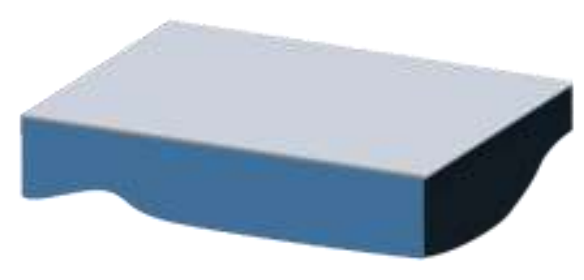

c)

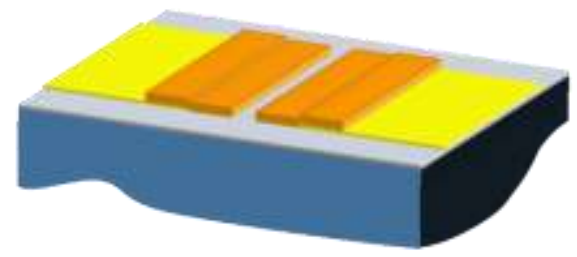

b)

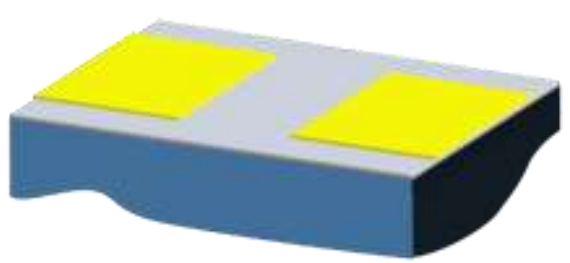

d)

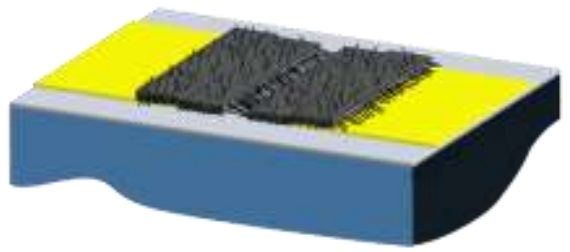

$\mathrm{Au} / \mathrm{Ti}$

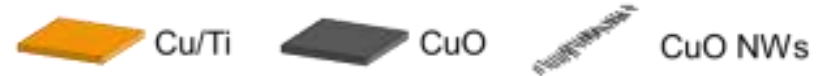

Figure S1. Diagram of the process of $\mathrm{CuO} \mathrm{NW}$ device fabrication. Lithography takes place on a) a (100) $\mathrm{Si}$ wafer substrate with a $300 \mathrm{~nm} \mathrm{SiO} 2$ insulating layer. b) An adhesion layer of $5 \mathrm{~nm}$ of $\mathrm{Ti}$ was deposited followed by a $200 \mathrm{~nm}$ layer of $\mathrm{Au}$ as electrical contacts. Another layer of $\mathrm{Ti} / \mathrm{Cu}(5$ $\mathrm{nm}$ and $1000 \mathrm{~nm}$, respectively) was deposited over part of the $\mathrm{Au}$ and $\mathrm{SiO}_{2}$ forming the structure shown in c). Finally, d) $\mathrm{CuO}$ NWs growth bridges the electrodes via thermal oxidation at $350^{\circ} \mathrm{C}$. 


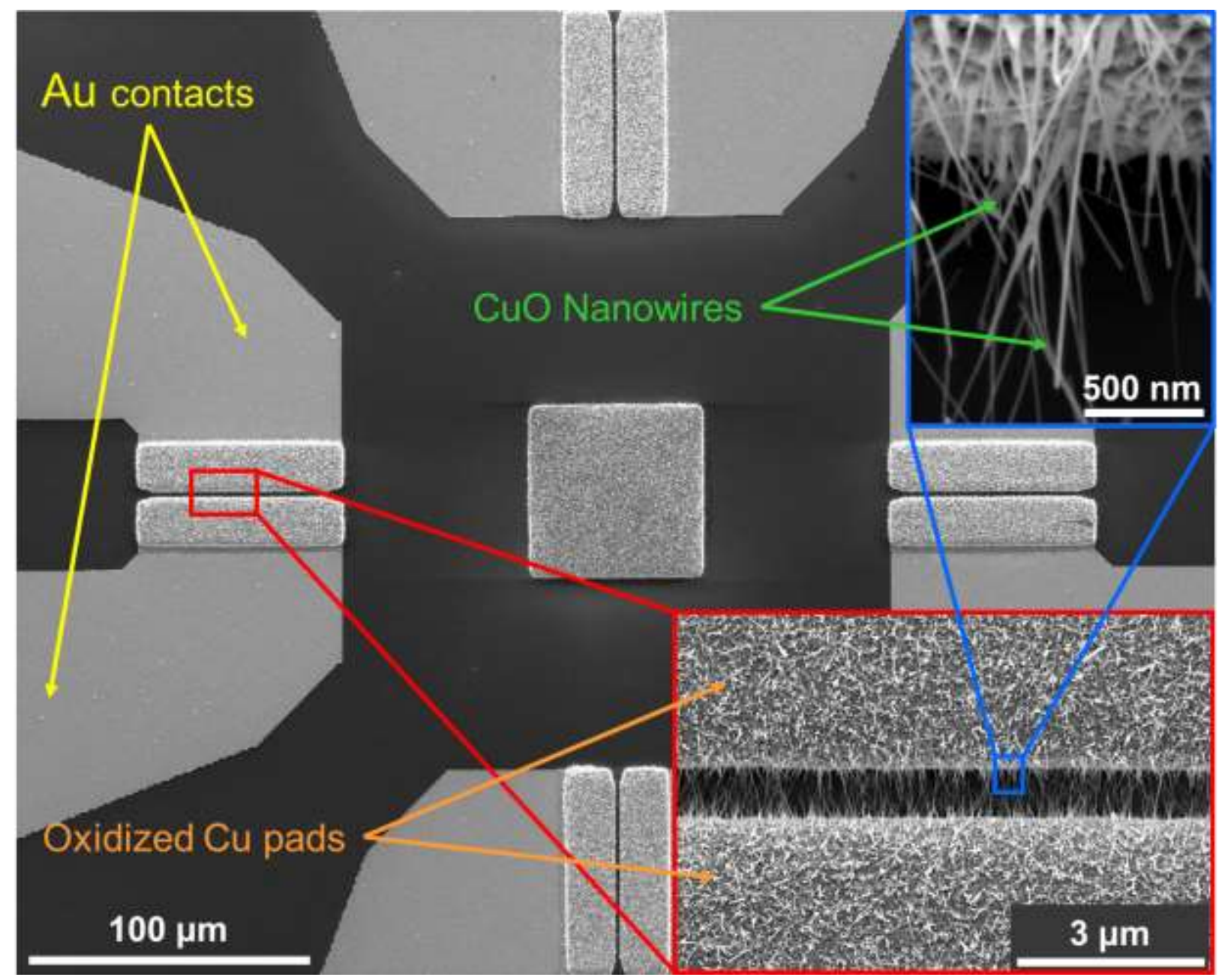

Figure S2. A scanning electron microscope (SEM) image showing the actual device. The red inset shows a close-up of the oxidized copper pads where the nanowires grow, while the blue inset shows the growing nanowire bridges comprising the active part of the device. 


\section{S2. Conductometric measurements of individual devices with conditions}

a)

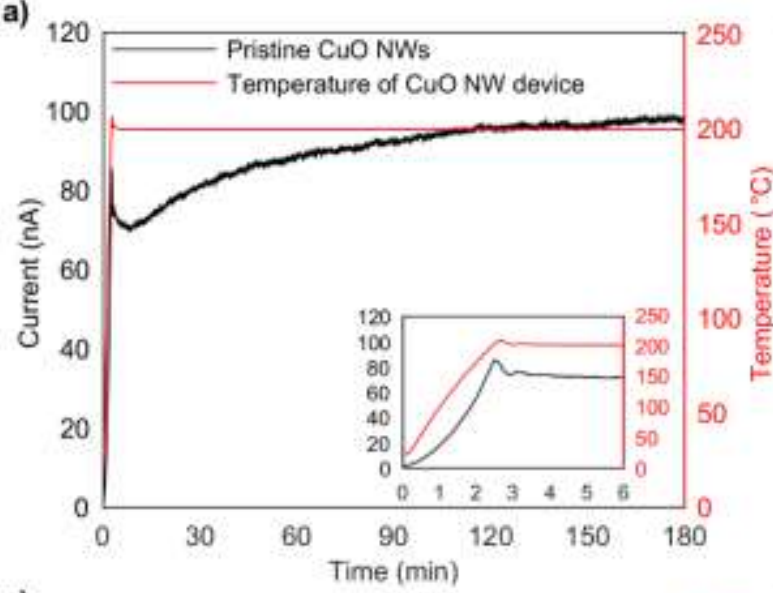

c)

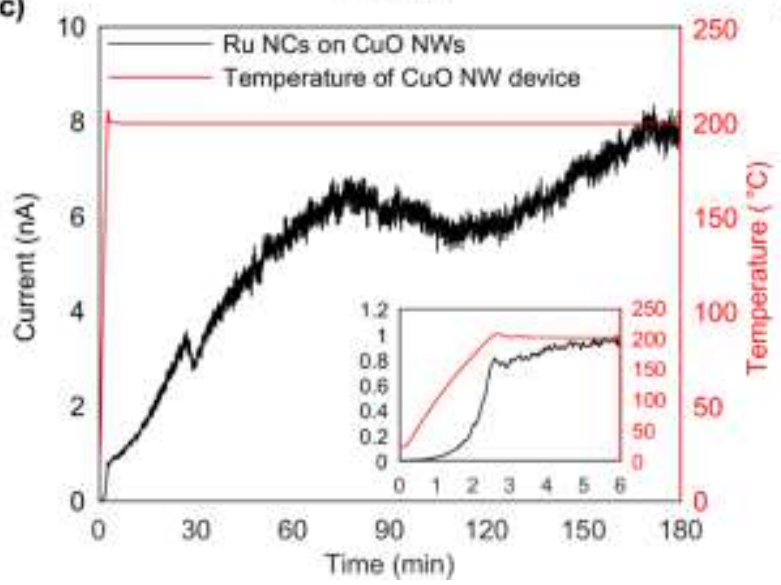

b)

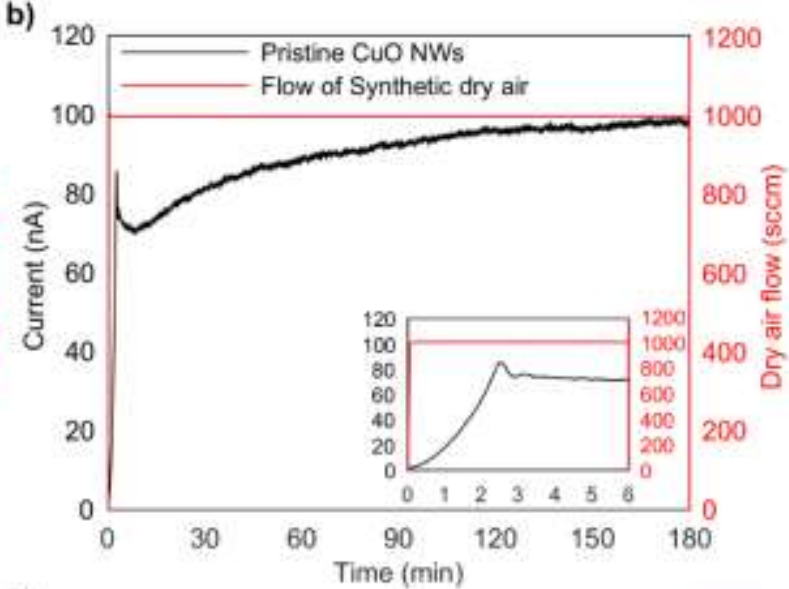

d)

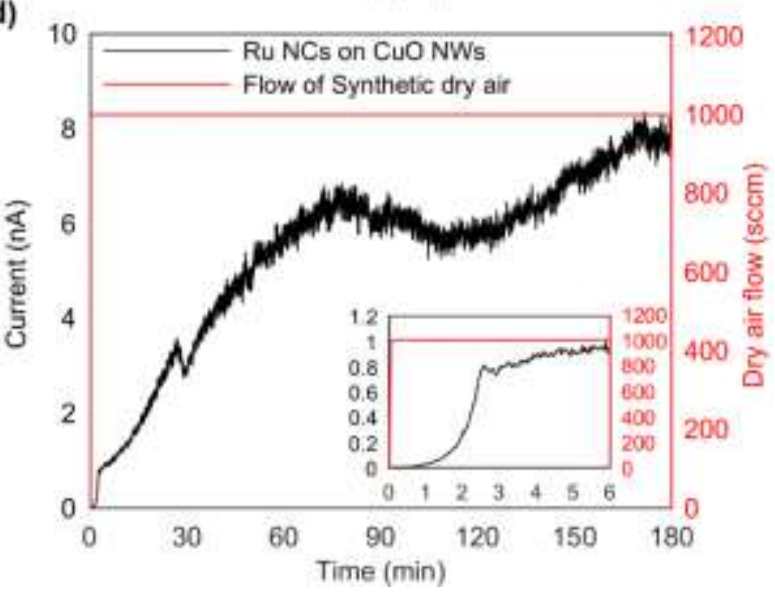

Figure S3. The current through the a) pristine and b) Ru-decorated $\mathrm{CuO} N W$ s and the temperature $\left(200^{\circ} \mathrm{C}\right)$ of the hotplate thermocouple. Similarly, the current through the nanowires is plotted along with the flow of dry air through the MFCs for the c) pristine and d) Ru-decorated devices. 
a)

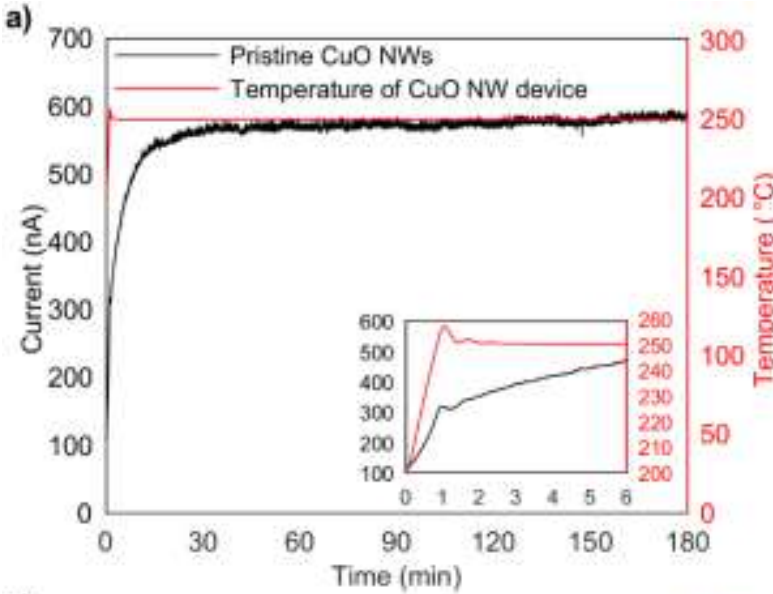

c)

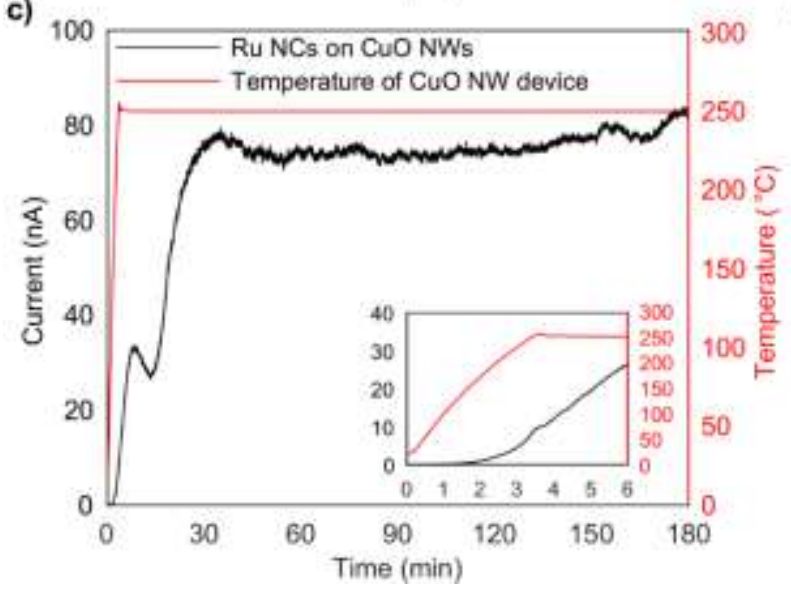

b)

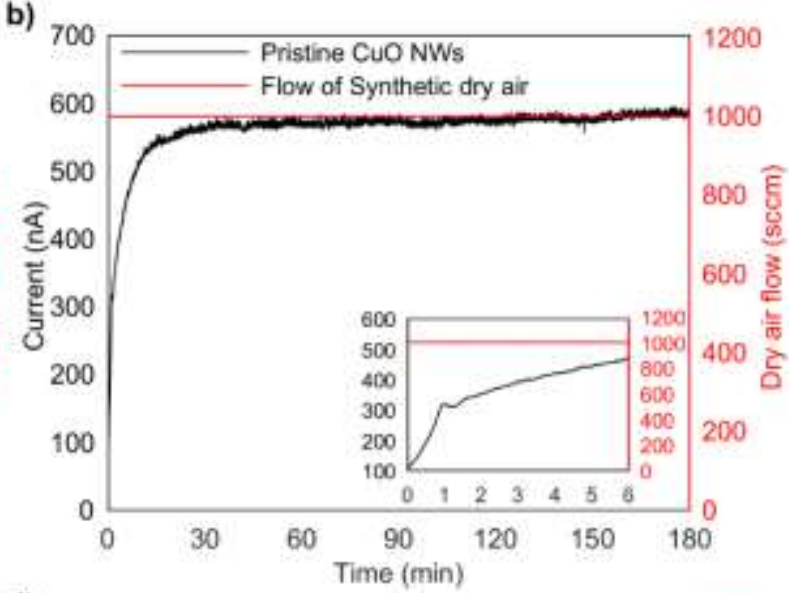

d)

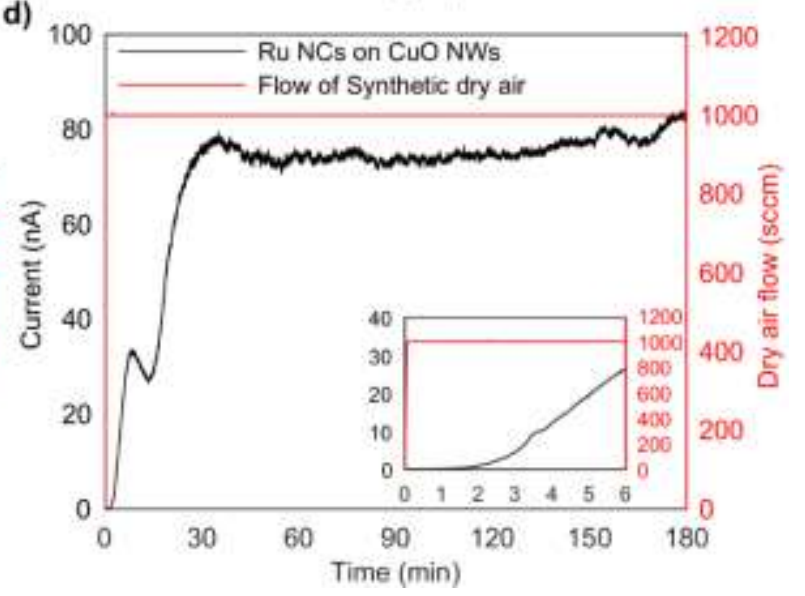

Figure S4. The current through the a) pristine and b) Ru-decorated $\mathrm{CuO}$ NWs and the temperature $\left(250^{\circ} \mathrm{C}\right)$ of the hotplate thermocouple. Similarly, the current through the nanowires is plotted along with the flow of dry air through the MFCs for the c) pristine and d) Ru-decorated devices. 


\section{S3. High resolution X-ray photoelectron spectroscopy (XPS)}
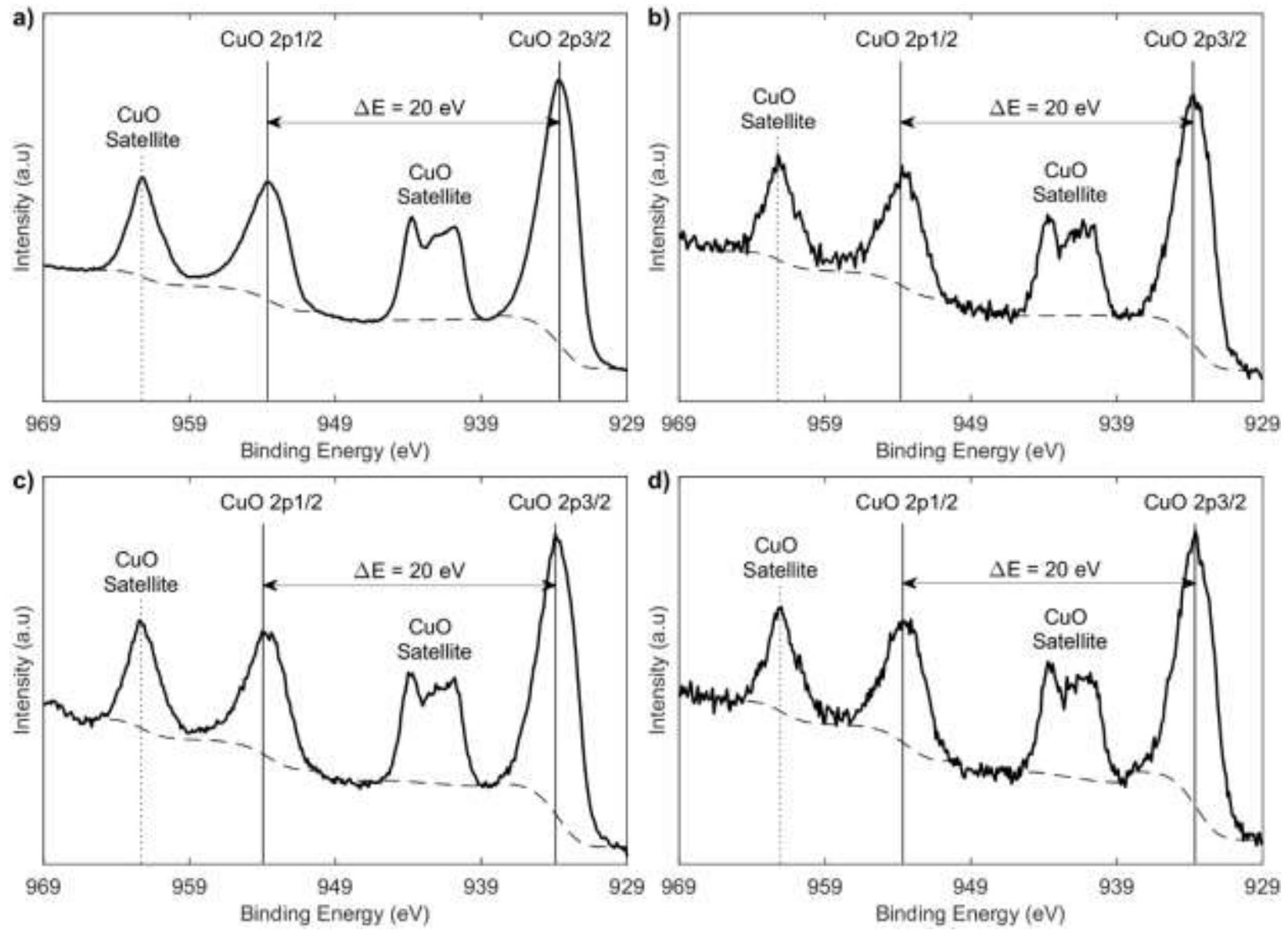

Figure S5. XPS spectra of $\mathrm{Cu} 2 \mathrm{p}$ of thermally oxidised $\mathrm{CuO}$ NWs a) pristine, b) with $\mathrm{Ru} \mathrm{NCs}$ as deposited, c) with Ru NCs after 1 hour of annealing in dry air and d) with Ru NCs after 5 hours of annealing in dry air. The difference between doublet peaks is indicated with $\Delta \mathrm{E}$, while doublets and satellites are labelled.

Figure $\mathbf{S 5}$ shows the XPS spectra of the $\mathrm{Cu} 2 \mathrm{p}$ of $\mathrm{CuO}$ NW from pristine to decorated with $\mathrm{Ru}$ and annealed for 5 hours. The main $\mathrm{Cu}$ peaks in all the images have a $\mathrm{Cu} 2 \mathrm{p} 3 / 2$ around $933.7 \mathrm{eV}$, which is consistent with $\mathrm{CuO} .{ }^{1,2}$ The split spin-orbit between $3 / 2$ and $1 / 2$ states is $20 \mathrm{eV}$ in all cases, again consistent with $\mathrm{CuO} .{ }^{1}$ The satellite peak between $939 \mathrm{eV}$ and $945 \mathrm{eV}$ has a shape and position characteristic of $\mathrm{CuO}$ as opposed to $\mathrm{Cu}_{2} \mathrm{O}$ or $\mathrm{Cu}(\mathrm{OH})_{2}{ }^{2}$ 
There appears to be no shift between the peaks of the $\mathrm{CuO}$. This adds further weight that the $\mathrm{C} 1 \mathrm{~s}$ peak position is being shifted due to the changes in the work function rather than chemical shifts due to the surface oxidation states of other chemical components. As with the $\mathrm{Ru} 3 \mathrm{~d}$ and $\mathrm{Cu} 2 \mathrm{p}$ peaks, the $\mathrm{O}$ 1s also shows no chemical shift, further evidencing the shift in the $\mathrm{C} 1 \mathrm{~s}$ positions.

The $\mathrm{O}$ 1s peak is difficult to deconvolute in a situation such as this. With $\mathrm{Ru}, \mathrm{Cu}$ and carbonic oxides being identified in the spectrum, it is difficult to identify which part off the $\mathrm{O} 1 \mathrm{~s}$ peak corresponds to which specific oxide. Therefore, in Figure S6, only obvious peaks relating to general lattice oxide (both from $\mathrm{Cu}$ and $\mathrm{Ru})^{2,3}$, defect oxide/metallic charge transfer $(\mathrm{Cu} / \mathrm{Ru})^{2,4}$ and carbonic oxides are identified. ${ }^{1}$ 

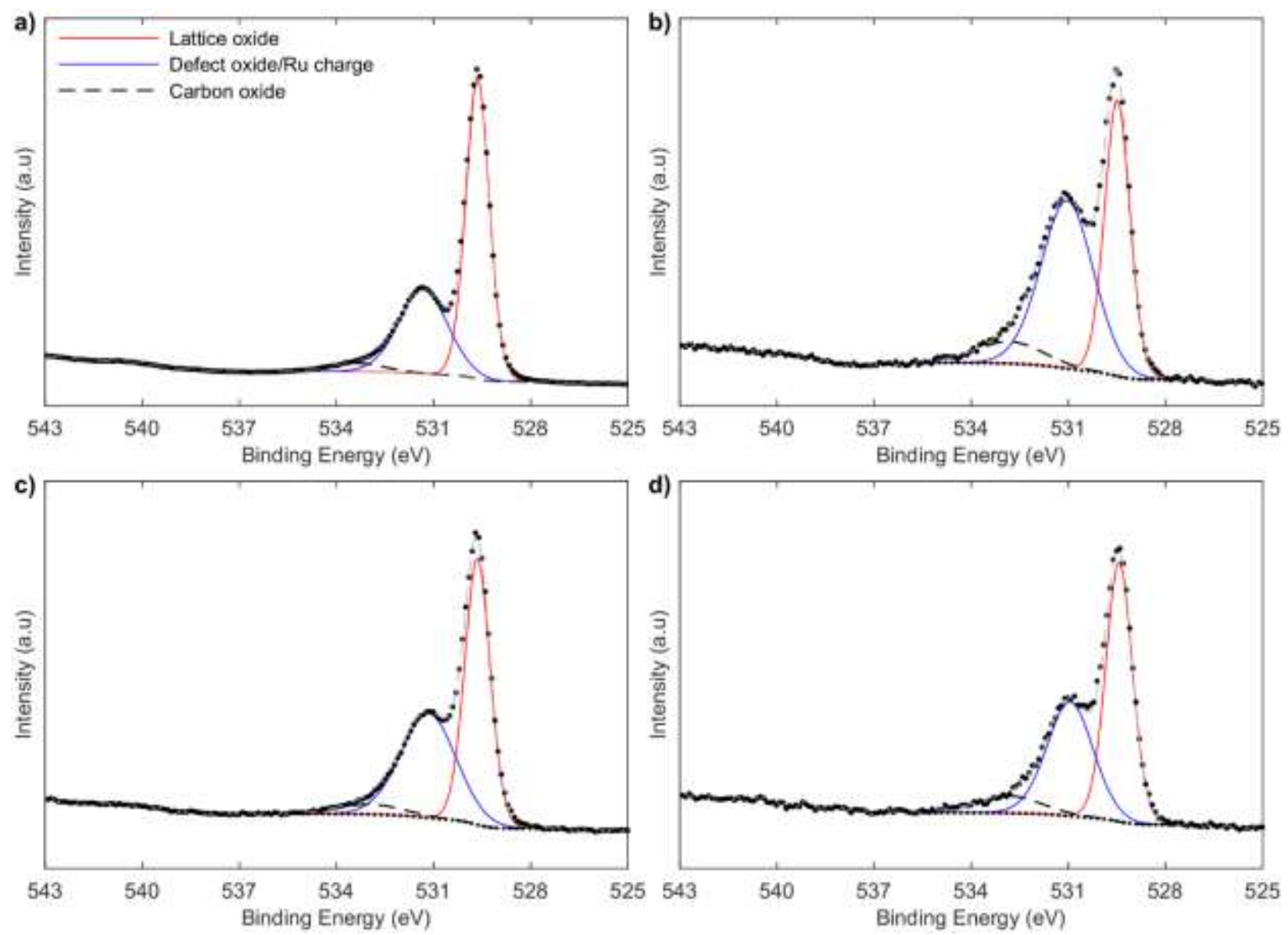

Figure S6. XPS spectra $(\bullet)$ of O 1s of thermally oxidised CuO NWs a) pristine, b) with Ru NCs as deposited, c) with Ru NCs after 1 hour of annealing in dry air and d) with Ru NCs after 5 hours of annealing in dry air. Lattice peak is represented using a red sub peak, defect oxide/Ru charge by a blue sub peak and carbonic oxide peaks shown as a black dotted sub-peak. Fitting envelope and background are indicated by the dashed grey and black lines, respectively.

The highest binding energies around $532.8 \mathrm{eV}$ are assigned to the carbonic oxides present. ${ }^{1,3}$ The $\mathrm{O}$ 1s peak of pristine and $\mathrm{Ru}$ decorated $\mathrm{CuO}$ can be seen in Figure S6. The peak position associated with metal oxide lattice are located at B.E. of $529.6 \mathrm{eV}$ and $529.5 \mathrm{eV}$ in each spectrum respectively. This is generally associated with the lattice oxide in $\mathrm{CuO}$ and $\mathrm{RuO}_{2 .}{ }^{2,3}$ The small peak next to this peak of $531.3 \mathrm{eV}$ is likely due to defect copper. ${ }^{2}$ Interestingly, this peak increases in concentration 
when Ru NCs are deposited. In addition to the $\mathrm{Cu}$ defect oxide peak, this position has also been associated with charge and electron transfer/ $d$-orbital readjustments in platinum group metals. ${ }^{3-5}$ Therefore, this could be experimental indication and support of the charge transfer suggested by the density functional theory (DFT) simulations. Interestingly, as the time of annealing increases, the peak area for this shoulder decreases, suggesting either a decrease in defects or a decrease in charge transfer. The latter seems likely when compared to the data shown by the PF-KPFM measurements, which show that annealed samples lack the charge transfer observed on as deposited samples. 


\section{S4. (Scanning) Transmission electron microscopy ((S)TEM) analysis of Ru NCs and Ru NCs on $\mathrm{CuO}$ NWs}
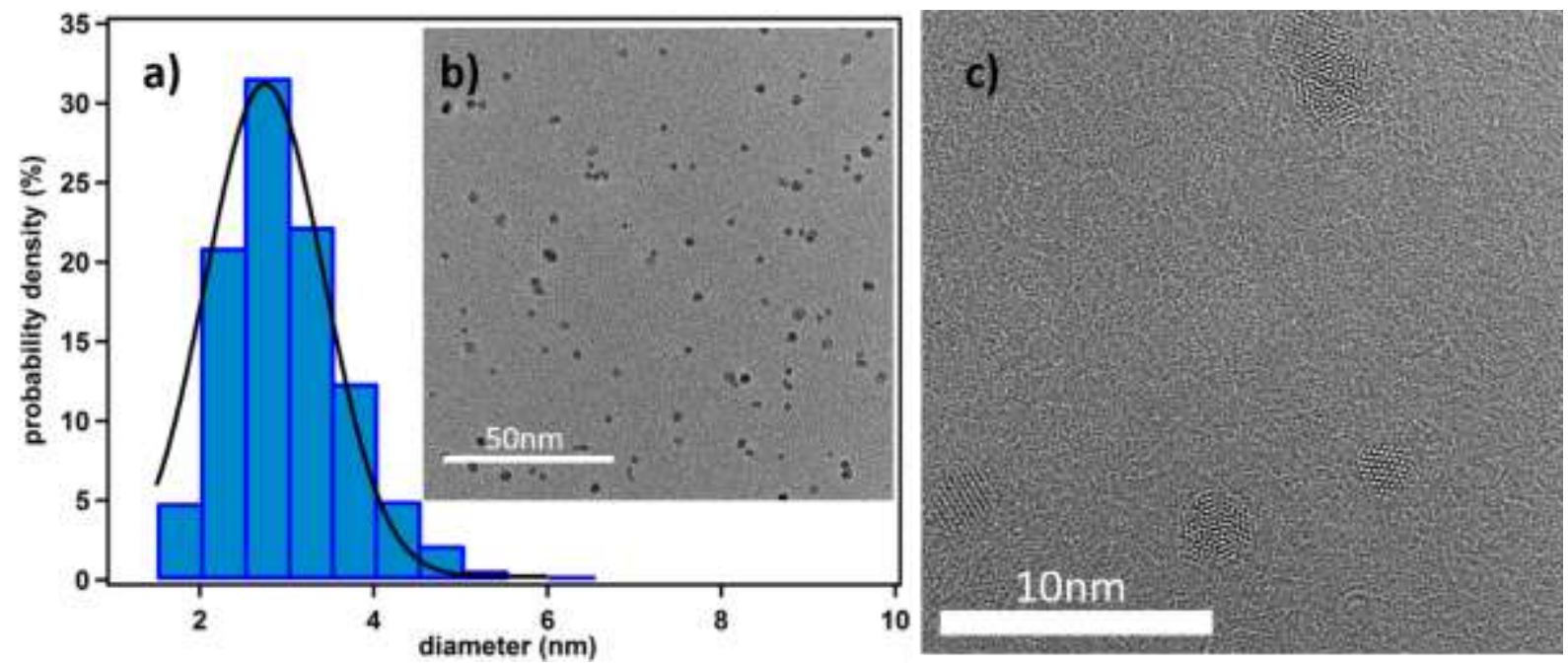

Figure S7. The a) size distribution of the deposited Ru NCs, b) an example of a low magnification TEM image used to calculate the size of the nanoclusters and $\mathbf{c}$ ) a medium magnification image showing single crystal hcp and icosahedral structure.

Figure $\mathbf{S} 7$ shows the size distribution of Ru NCs. Samples were calculated from approximately 800 clusters across ten (10) snapshots of a low density (deposition time of 8 minutes) sample. The sizes were calculated using the program ImageJ on low magnification images, an example of which is shown in Figure S7. The samples were blurred using the Accurate Gaussian Blur plugin with a blur of 2.0. This was to remove the detail of the amorphous carbon grid background, creating a better contrast between the clusters (dark) and the background (light). The threshold function was used to create a black and white image, which was then used to estimate the area of nanoclusters, using the particles function built into ImageJ. Circularity was set from $0-1$, while the size of the clusters was limited from 1 to infinity $\mathrm{nm}^{2}$ areas. Images on the edges were not considered as the full area is not known. Once the areas were determined, an estimation of the nanocluster diameter 
was made, assuming the clusters were spherical using the rearranged formula for area of a circle seen in Equation S1.

$d=2 \sqrt{\frac{A}{\pi}}$

Where $d$ is the approximate diameter of the nanocluster, and $A$ is the area calculated from ImageJ.

Finally, Figure S7 shows a medium magnification image of the sample highlighting both icosahedral and single crystal structured nanoclusters. This is an example of the magnification used to investigate the ratio of icosahedral and single crystal nanoclusters. 

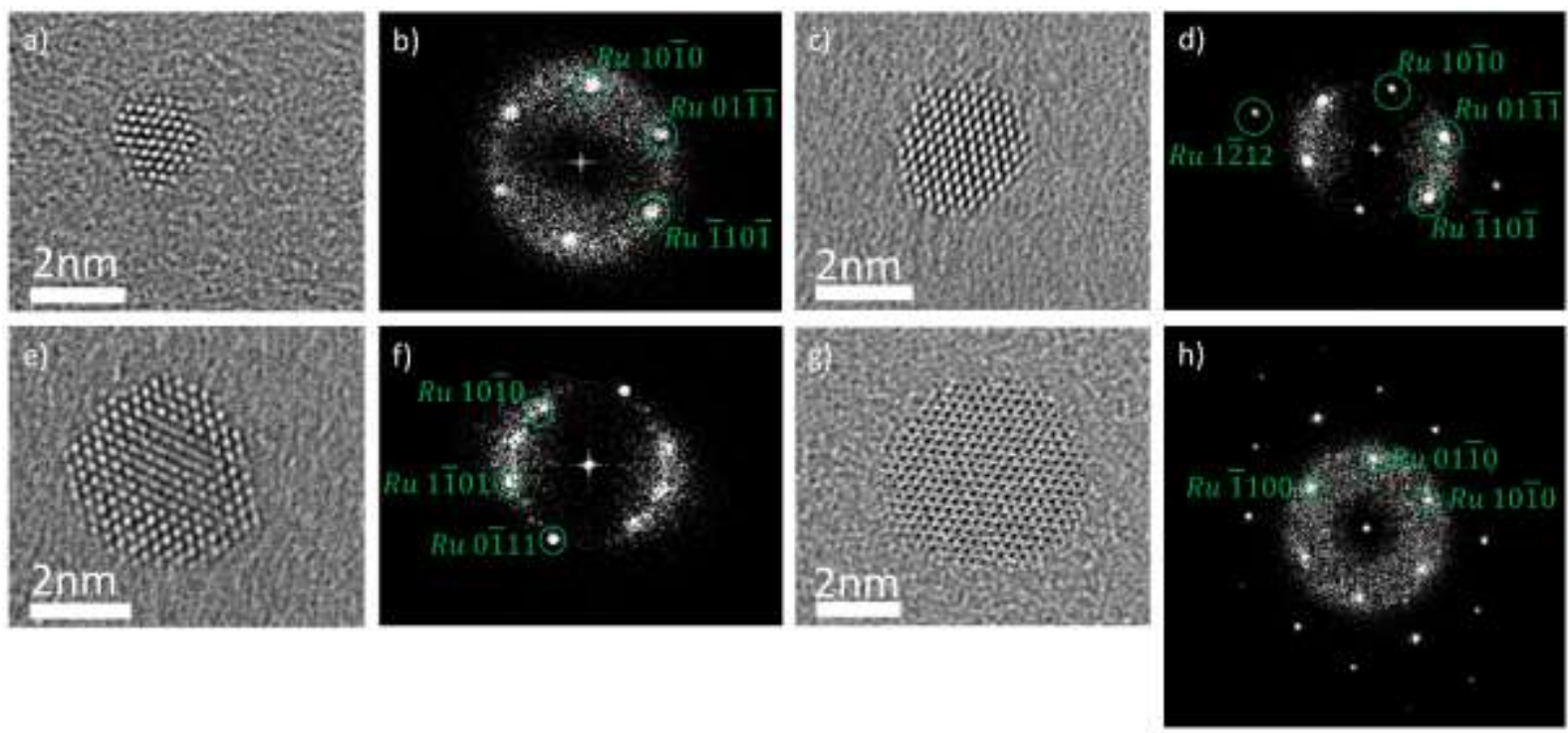

Figure S8. TEM images of different sizes of single crystal Ru NCs on amorphous carbon TEM grids a), c), e) and g). Fast Fourier transforms (FFT) of each respective image can be seen from b), d), f) and h), identifying visible lattice planes. Images in a), c) and e) are seen imaged on zone axis [1릴

Figure S8 shows TEM images of single crystal Ru NCs visualized on different zone axes with different sizes as deposited on a carbon grid. These conditions should show the nanoclusters crystal lattice in the most relaxed deposited state possible, as there is less cluster-substrate interaction (amorphous carbon substrate). The two main zone axes observed are hexagonal closed packed $([0001] \mathbf{g})$ and $[\overline{1} 2 \overline{1} 3] \mathbf{a}), \mathbf{c})$ and $\mathbf{e})$ ). This shows the facets of the $\{10 \overline{1} 0\}$ family (on both axes) and $\{1 \overline{1} 01\}$ family in the $[\overline{1} 2 \overline{1} 3]$ case. The clusters appear to have minor variations in lattice spacings based on size (summarized in Table S1). The smaller clusters show exaggerated sizes in the (1101) family, likely due to the higher stress created by more dangling bonds at the surface. As the surface area decreases in the larger clusters, the stress subsequently decreases leaving the larger clusters closer to the bulk value. 
Table S1. The lattice $d$-spacings of single crystal Ru NCs for the nanoclusters seen in Figure S8.

\begin{tabular}{|c|c|c|c|c|}
\hline $\begin{array}{l}\text { Size of } \\
\text { particle } \\
(\mathrm{nm})\end{array}$ & $\begin{array}{c}\text { Ru zone axis } \\
\text { observed }\end{array}$ & $\begin{array}{l}\text { Ru lattice } \\
\text { plane }\end{array}$ & $\begin{array}{c}\text { Lattice } \\
\text { spacing } \\
\text { (nm) }\end{array}$ & $\begin{array}{c}\text { Theoretical } \\
\text { lattice spacing } \\
(\mathrm{nm})^{6-8}\end{array}$ \\
\hline \multirow[t]{3}{*}{$1.75(a)$} & $\overline{1} 2 \overline{1} 3$ & $01 \overline{11}$ & 0.211 & 0.206 \\
\hline & & $\overline{1} 10 \overline{1}$ & 0.210 & 0.206 \\
\hline & & $10 \overline{1} 0$ & 0.231 & 0.234 \\
\hline \multirow[t]{4}{*}{$2.97(\mathrm{c})$} & $\overline{1} 2 \overline{1} 3$ & $01 \overline{11}$ & 0.209 & 0.206 \\
\hline & & $\overline{1} 10 \overline{1}$ & 0.208 & 0.206 \\
\hline & & $10 \overline{1} 0$ & 0.238 & 0.234 \\
\hline & & $1 \overline{2} 12$ & 0.116 & 0.114 \\
\hline \multirow[t]{3}{*}{$3.16(\mathrm{e})$} & $\overline{1} 2 \overline{1} 3$ & $0 \overline{1} 11$ & 0.207 & 0.206 \\
\hline & & $1 \overline{1} 01$ & 0.205 & 0.206 \\
\hline & & $10 \overline{1} 0$ & 0.234 & 0.234 \\
\hline \multirow[t]{3}{*}{$4.64(\mathrm{~g})$} & 0001 & $\overline{1} 100$ & 0.234 & 0.234 \\
\hline & & $01 \overline{1} 0$ & 0.234 & 0.234 \\
\hline & & $10 \overline{1} 0$ & 0.236 & 0.234 \\
\hline
\end{tabular}




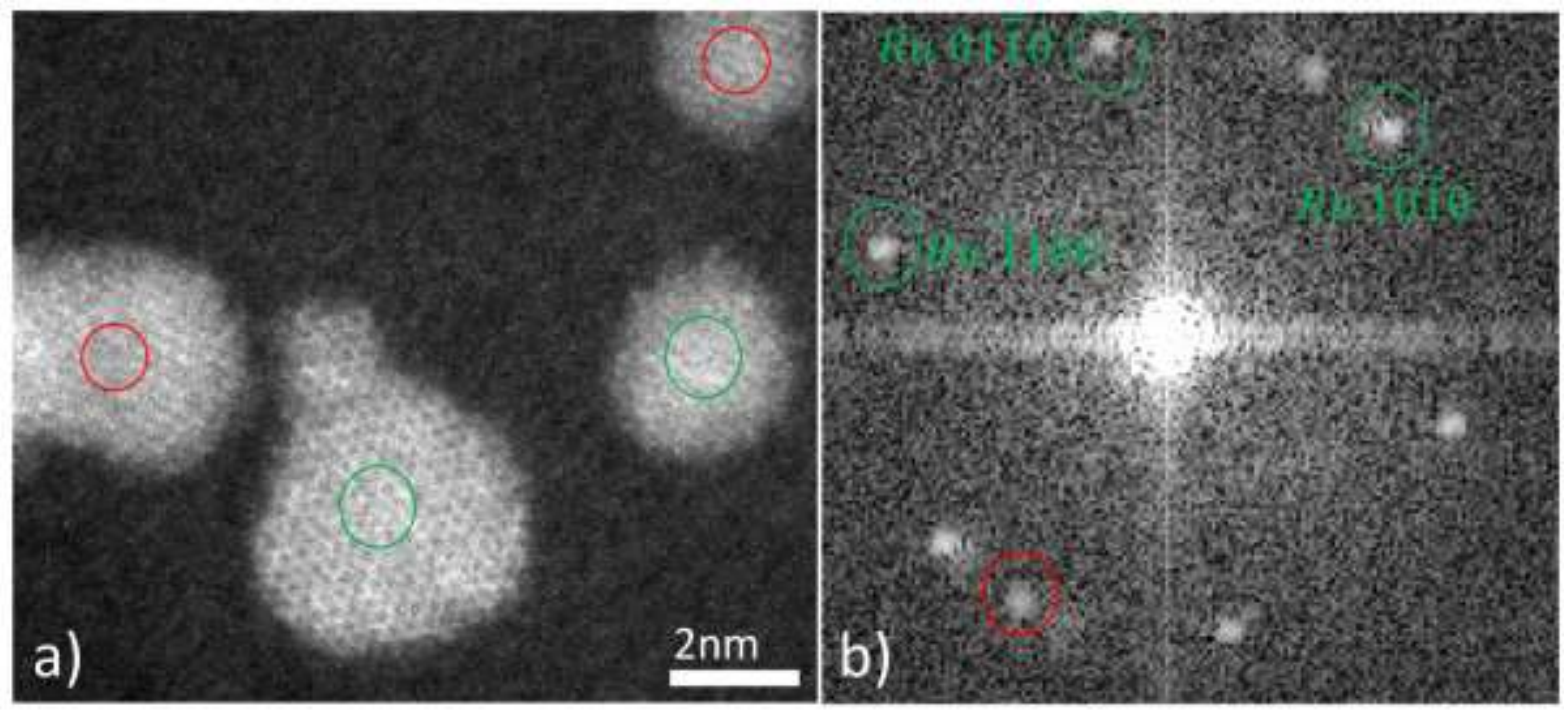

Figure S9. a) A STEM image of single crystal Ru NCs on an amorphous carbon grid, and b) the corresponding FFT. Cluster on zone axis appears on [0001] as determined from the plane lengths and angles between the planes (FFT spots). Green and red circles indicate which FFT spots are related to each cluster.

Figure S9 shows images of similar magnification taken using Cs probe corrected STEM (JEOL ARM200). The image shows one cluster on zone axis, while other clusters show one or fewer planes. The full FFT is also shown. Almost all these clusters correspond to lattice spots which is seen in the cluster on zone axis [0001], except for the far left and top right clusters which correspond to the lattice spot circled in red. All these spots correspond to the $\{10 \overline{1} 0\}$ family of planes and all are slightly larger than expected as surmised in Table S2. In these observations, there appears to be some discrepancy with regards to the Cs corrected TEM images. The overall trend of the TEM data is replicated (i.e. the $d$-spacings are of similar distances) indicating that the instrument is well aligned, but there is a small offset between the measured distances using the STEM mode. This suggests that the smaller $d$-spacings apparent in the nanoclusters on the nanowire are significant. 
Table S2. The lattice $d$-spacings of single crystal Ru NCs seen in Figure S9.

\begin{tabular}{cc}
\hline Ru lattice direction [0001] axis & $\begin{array}{c}d \text {-spacing } \\
(\mathrm{nm})\end{array}$ \\
\hline $10 \overline{1} 0$ & 0.239 \\
$01 \overline{1} 0$ & 0.240 \\
$\overline{1} 100$ & 0.241 \\
$10 \overline{1} 0$ plane family from \\
clusters circled in red
\end{tabular}


Table S3. The lattice $d$-spacings of single crystal Ru NCs on CuO NWs, along a variety of zone axes.

\begin{tabular}{|c|c|c|}
\hline Zone Axis & Ru Plane & $\begin{array}{c}d \text {-spacing } \\
\text { (nm) }\end{array}$ \\
\hline \multirow[t]{3}{*}{ [0001] } & $\overline{2} 200$ & 0.108 \\
\hline & $0 \overline{2} 20$ & 0.115 \\
\hline & $\overline{2} 020$ & 0.109 \\
\hline \multirow[t]{3}{*}[\overline{1}2\overline{1}3]{} & $0 \overline{1} 11$ & 0.195 \\
\hline & $1 \overline{1} 01$ & 0.193 \\
\hline & $10 \overline{1} 0$ & 0.227 \\
\hline \multirow[t]{3}{*}{ [011̄1] } & $1 \overline{1} 0 \overline{1}$ & 0.191 \\
\hline & $2 \overline{110}$ & 0.122 \\
\hline & $10 \overline{11}$ & 0.188 \\
\hline \multirow[t]{3}{*}{ [011̄2] } & $01 \overline{11}$ & 0.187 \\
\hline & $20 \overline{21}$ & 0.103 \\
\hline & $2 \overline{110}$ & 0.127 \\
\hline
\end{tabular}



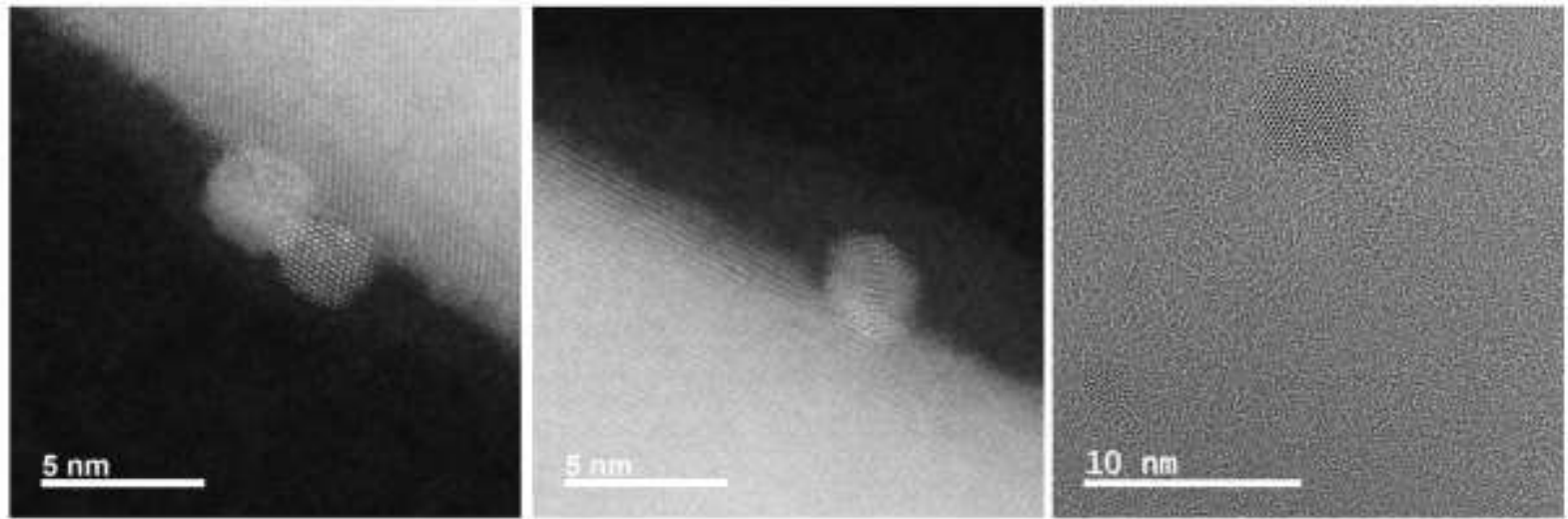

Figure S10. Full images of Figure 3.

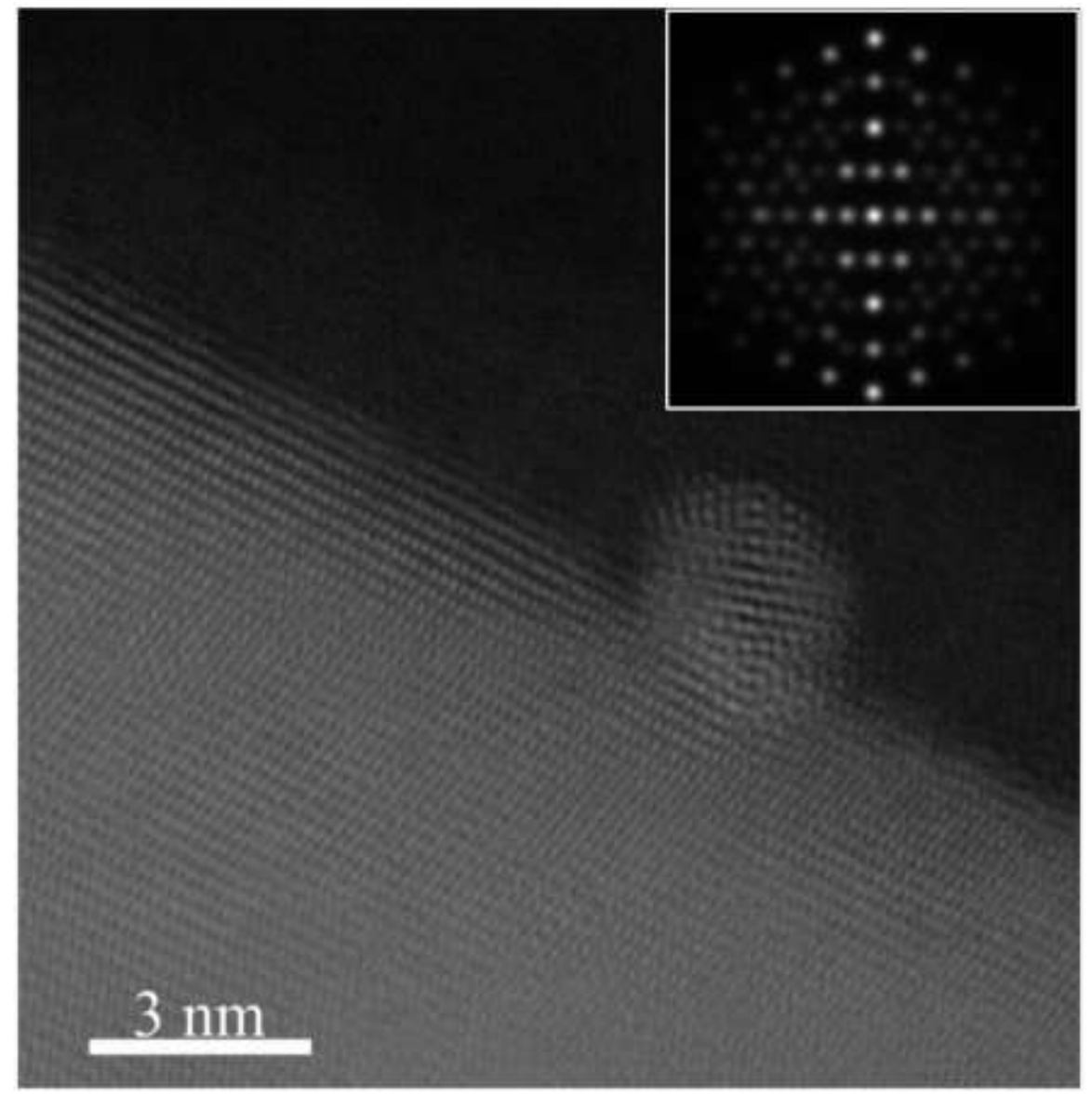

Figure S11. Filtered experimental high-angle annular dark-field scanning transmission electron microscopy (HAADF-STEM) image, while the inset shows a simulated image of the icosahedral fcc Ru-NC on 2-fold symmetry axis calculated by the EMS-JEMS software. ${ }^{9}$ 


\section{S5. STEM observation of nanocluster-nanowire system during transient periods}
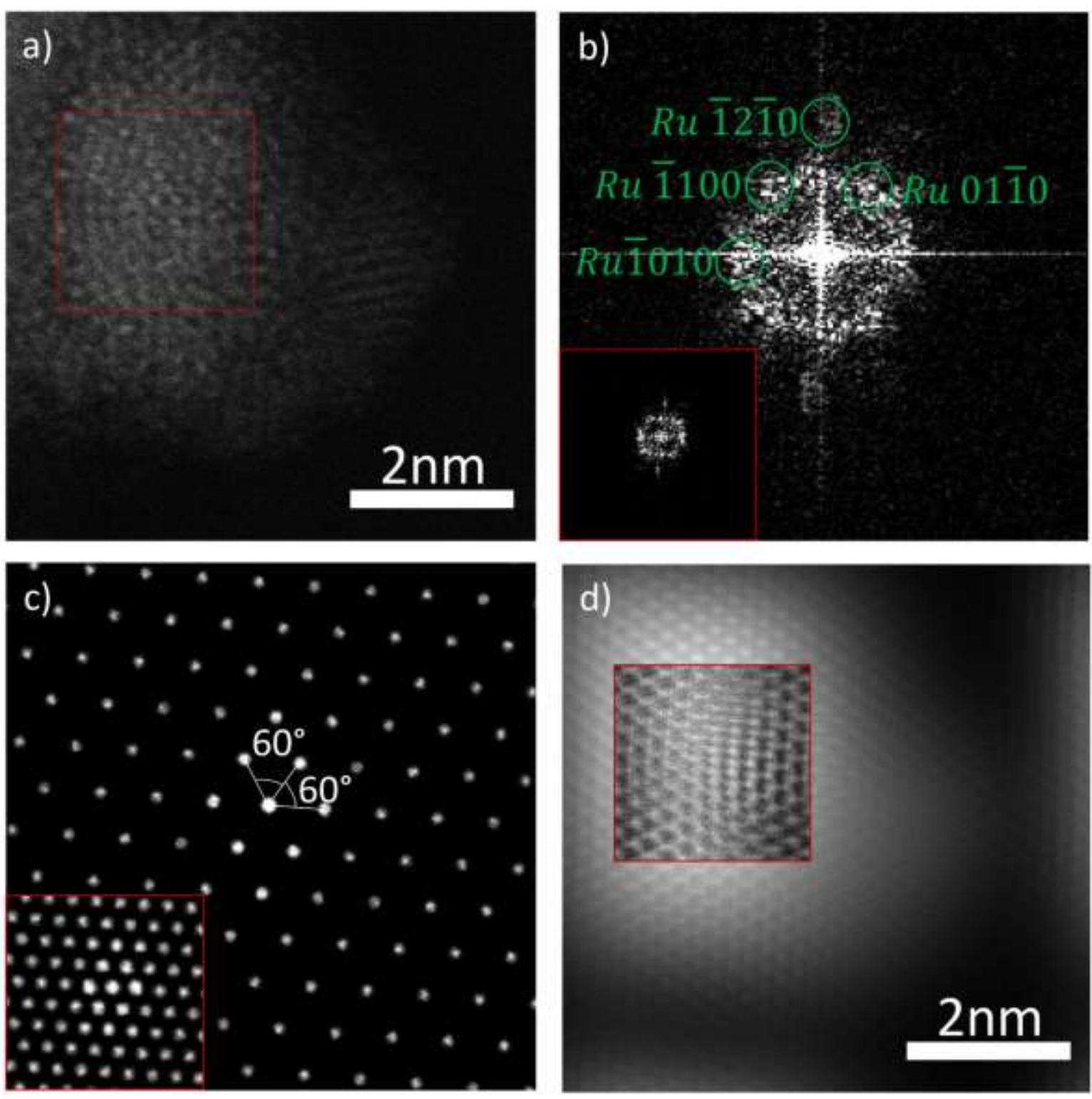

Figure S12. a) STEM-HAADF image of a $\mathrm{Ru} \mathrm{NC}$ on a $\mathrm{CuO} \mathrm{NW}$ after $e x$-situ annealing at $200^{\circ} \mathrm{C}$ for $45 \mathrm{~min}$ in dry air. b) Shows an FFT of the area shown in a). The inset FFT is from the area bounded by the red box in a). c) Is an array mask based on keeping a hexagonal pattern with $60^{\circ}$ angular separation between the inner most masking spots. The inset mask was created based on the inset FFT in b). d) Shows the resulting Bragg filtered image from c). The inset is based on the inset mask in c). 

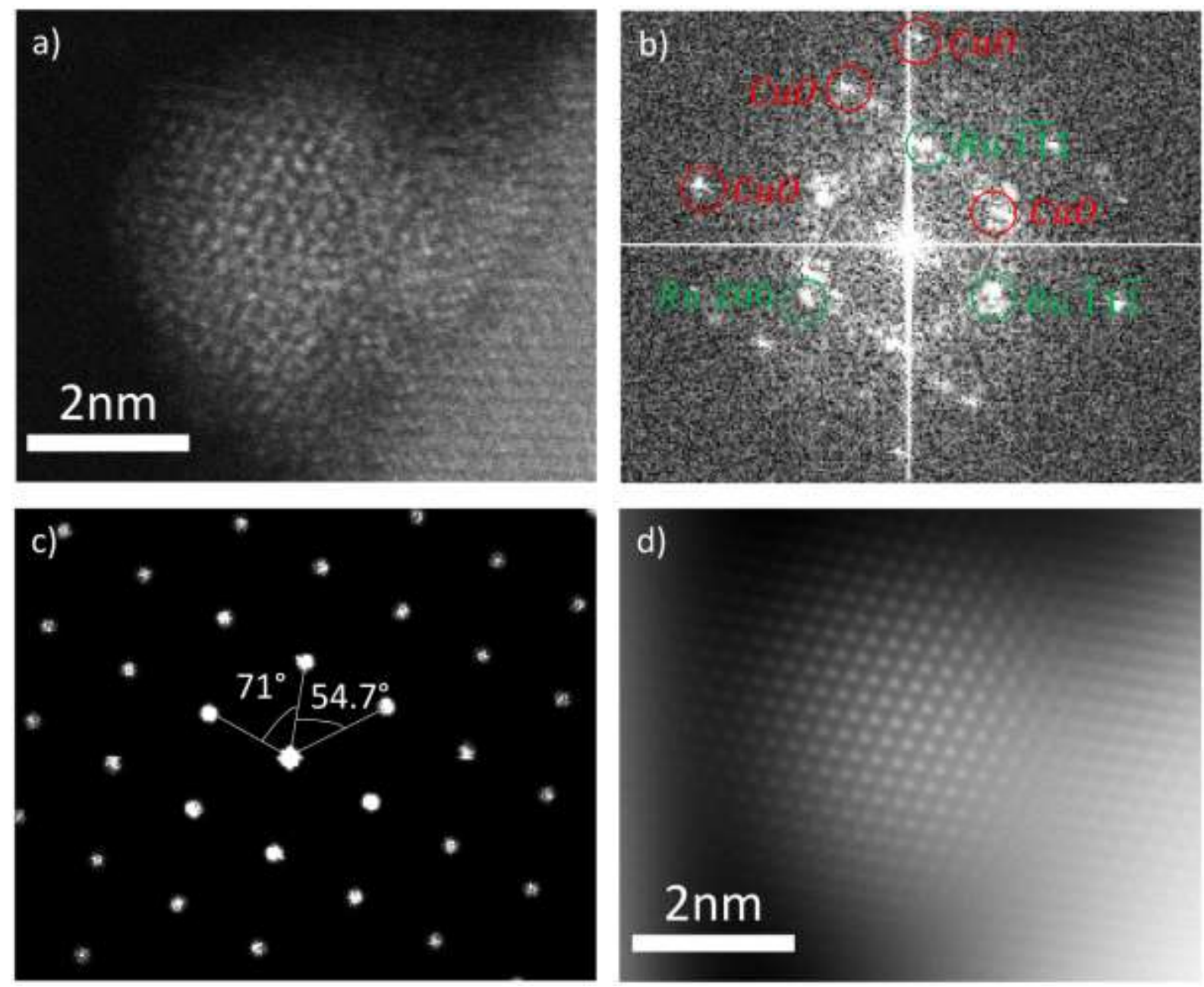

Figure S13. a) STEM-HAADF image of a Ru NC on a $\mathrm{CuO} \mathrm{NW}$ after ex-situ annealing at $200^{\circ} \mathrm{C}$ for 66 min in dry air. b) Shows an FFT of the area shown in a). c) An array mask based on keeping an almost hexagonal pattern (fcc on [011]) with $54^{\circ}-71^{\circ}$ angular separation between the inner most masking spots. d) Shows the resulting Bragg filtered image from c). 

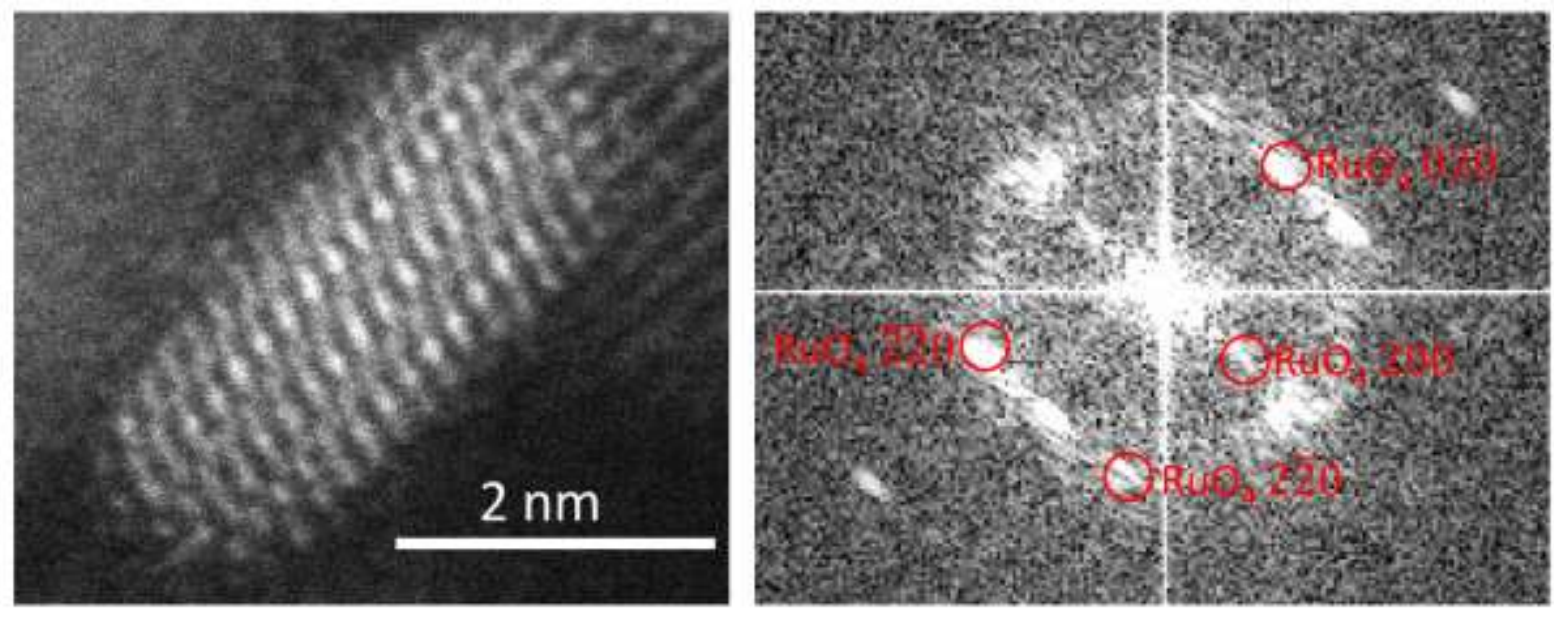

Figure S14. a) STEM-HAADF image of $\mathrm{RuO}_{4} \mathrm{NC}$ on a $\mathrm{CuO} \mathrm{NW}$ seen along the [001] direction. The structure of $\mathrm{RuO}_{4}$ has been determined as either a cubic ( $\left.P \overline{4} 3 n(218), a=0.851 \mathrm{~nm}\right)$ or monoclinic $\left(\mathrm{C} 2 / c(15), a=0.93 \mathrm{~nm}, b=0.44 \mathrm{~nm}, c=0.85 \mathrm{~nm}, \beta=116.82^{\circ}\right)$ crystal system. $\left.{ }^{10} \mathbf{b}\right)$ Shows an FFT confirming that the $\mathrm{RuO}_{4} \mathrm{NC}$ has crystallised in a monoclinic structure.
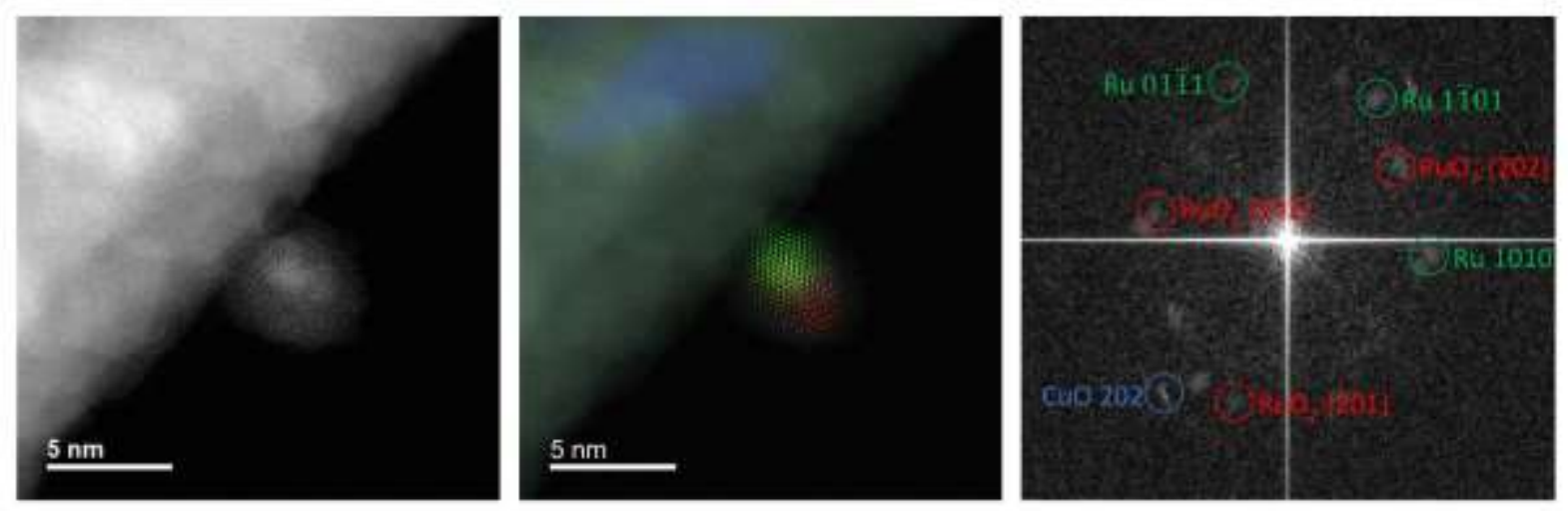

Figure S15. a) STEM-HAADF image of $\mathrm{RuO}_{2} @ \mathrm{Ru} \mathrm{NC}$ on a $\mathrm{CuO} \mathrm{NW}$ seen along the [0001] direction. b) A false colour image showing the presence of metallic $\mathrm{Ru}$ (green), $\mathrm{RuO}_{2}$ (red) and $\mathrm{CuO}$ (202) (blue) in the original image. c) Shows an FFT confirming the existence of a metallic $\mathrm{Ru}$ core and a tetragonal $\mathrm{RuO}_{2}$ shell. 

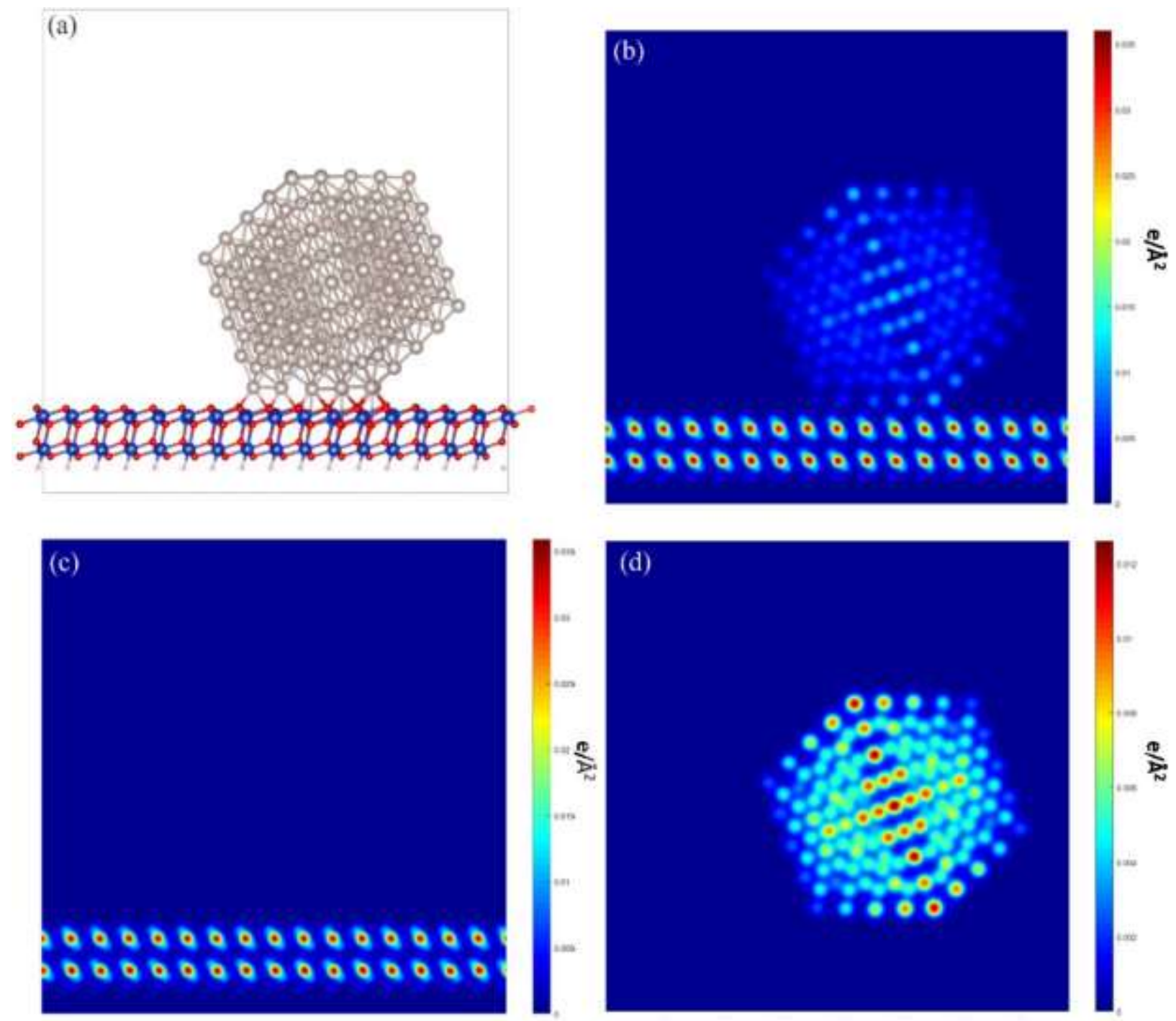

Figure S16. a) The relaxed atomistic model of the icosahedral $\mathrm{Ru} \mathrm{NC}$ on $\mathrm{CuO}$ surface along the projection direction of Figure 3. b) Shows the charge density projection of the model (seen in a), while c) highlights the charge density projection of the bare $\mathrm{CuO}$ surface and d) presents the charge density projection of the isolated icosahedral fcc Ru NC. 

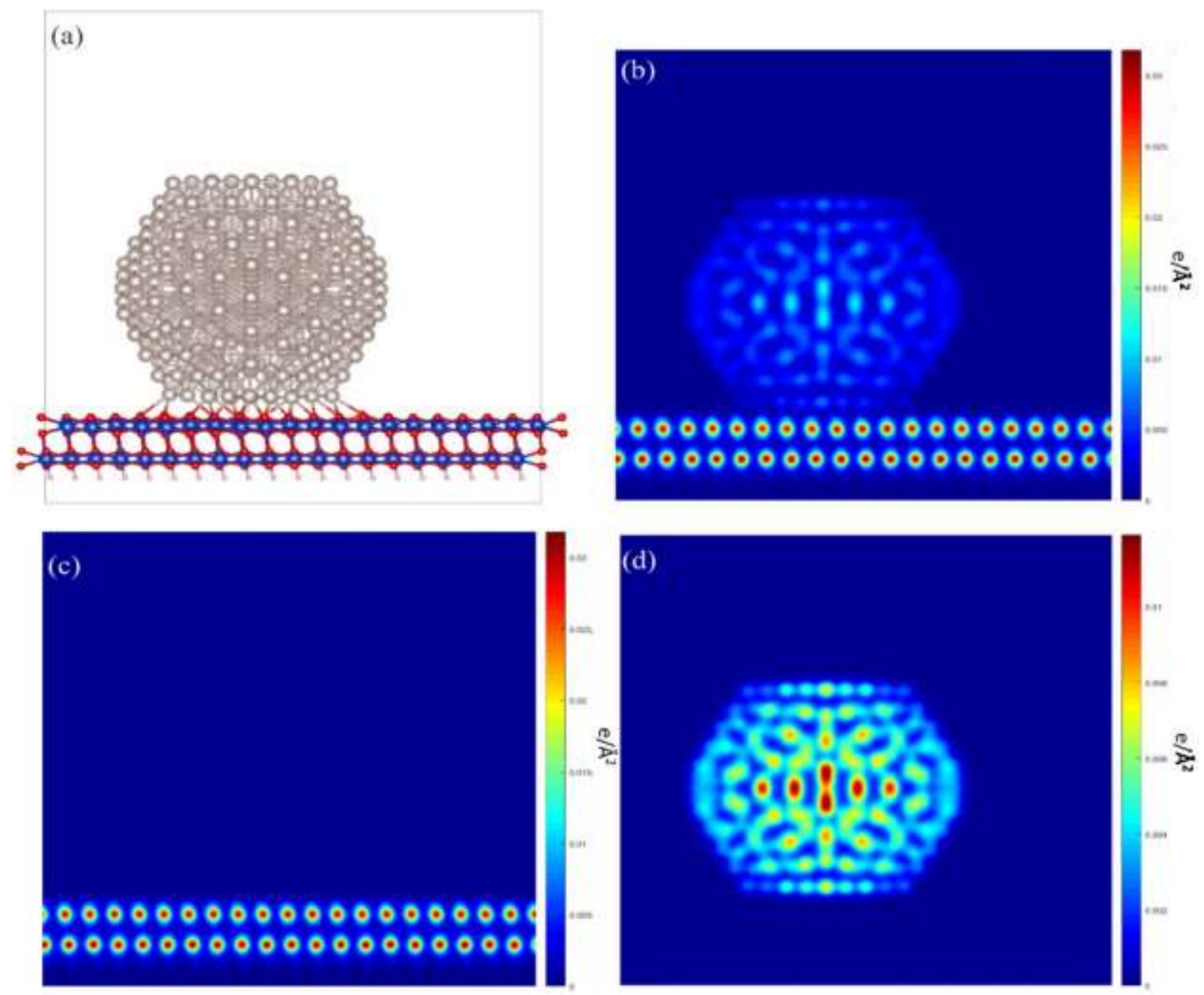

Figure S17. a) The relaxed atomistic model of the icosahedral $\mathrm{Ru} \mathrm{NC}$ on $\mathrm{CuO}$ surface along the perpendicular direction of Figure 3. b) Shows the charge density projection of the model (seen in a)), while c) highlights the charge density projection of the bare $\mathrm{CuO}$ surface and d) presents the charge density projection of the isolated icosahedral fcc Ru NC. 

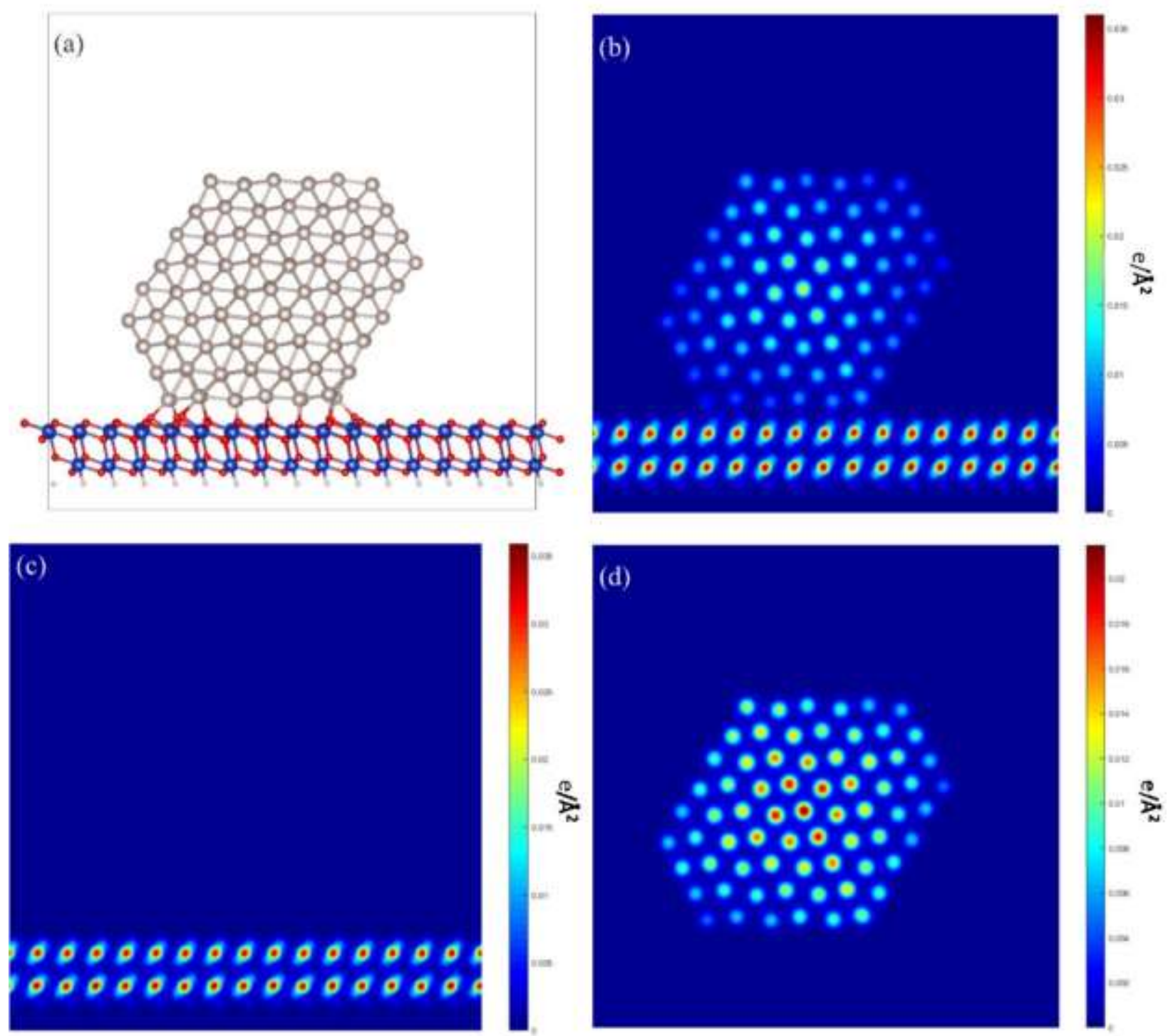

Figure S18. a) The relaxed atomistic model of the hcp $\mathrm{Ru} \mathrm{NP}$ on $\mathrm{CuO}$ surface along the along the [1120] direction. b) Shows the charge density projection of the model (seen in a)), while c) highlights the charge density projection of the bare $\mathrm{CuO}$ surface and d) presents the charge density projection of the isolated hcp $\mathrm{Ru} \mathrm{NC}$. 

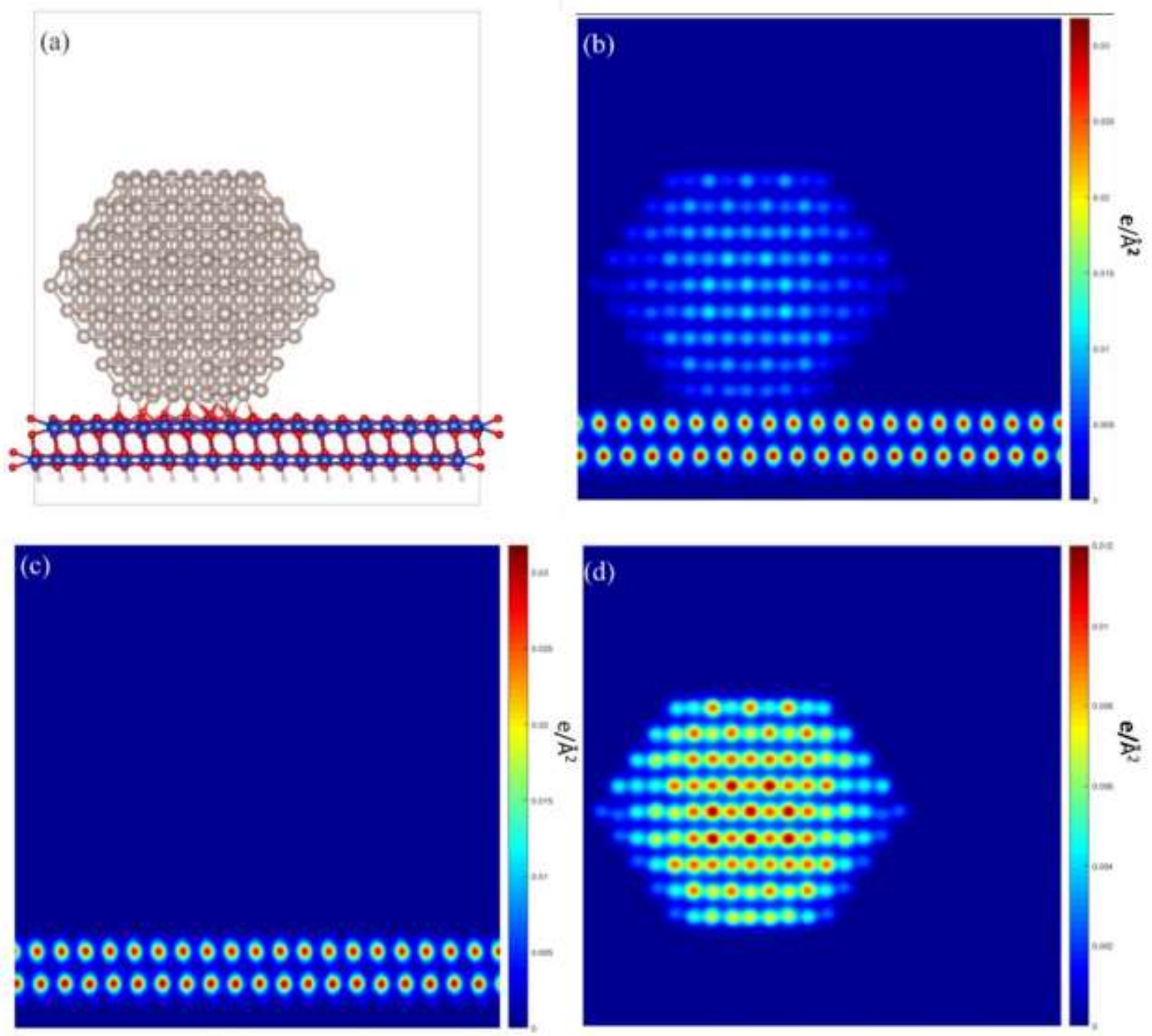

Figure S19 a) The relaxed atomistic model of the hcp Ru NP on CuO surface along the perpendicular ([10 $\overline{1} 0])$ direction to that seen in Figure S18. b) Shows the charge density projection of the model (seen in a)), while c) highlights the charge density projection of the bare $\mathrm{CuO}$ surface and $\mathbf{d})$ presents the charge density projection of the isolated hcp $\mathrm{Ru} \mathrm{NC}$. 
S7. PeakForce Kelvin probe force microscopy (PF-KPFM) supplement and $\mathrm{CuO}$ NW analysis

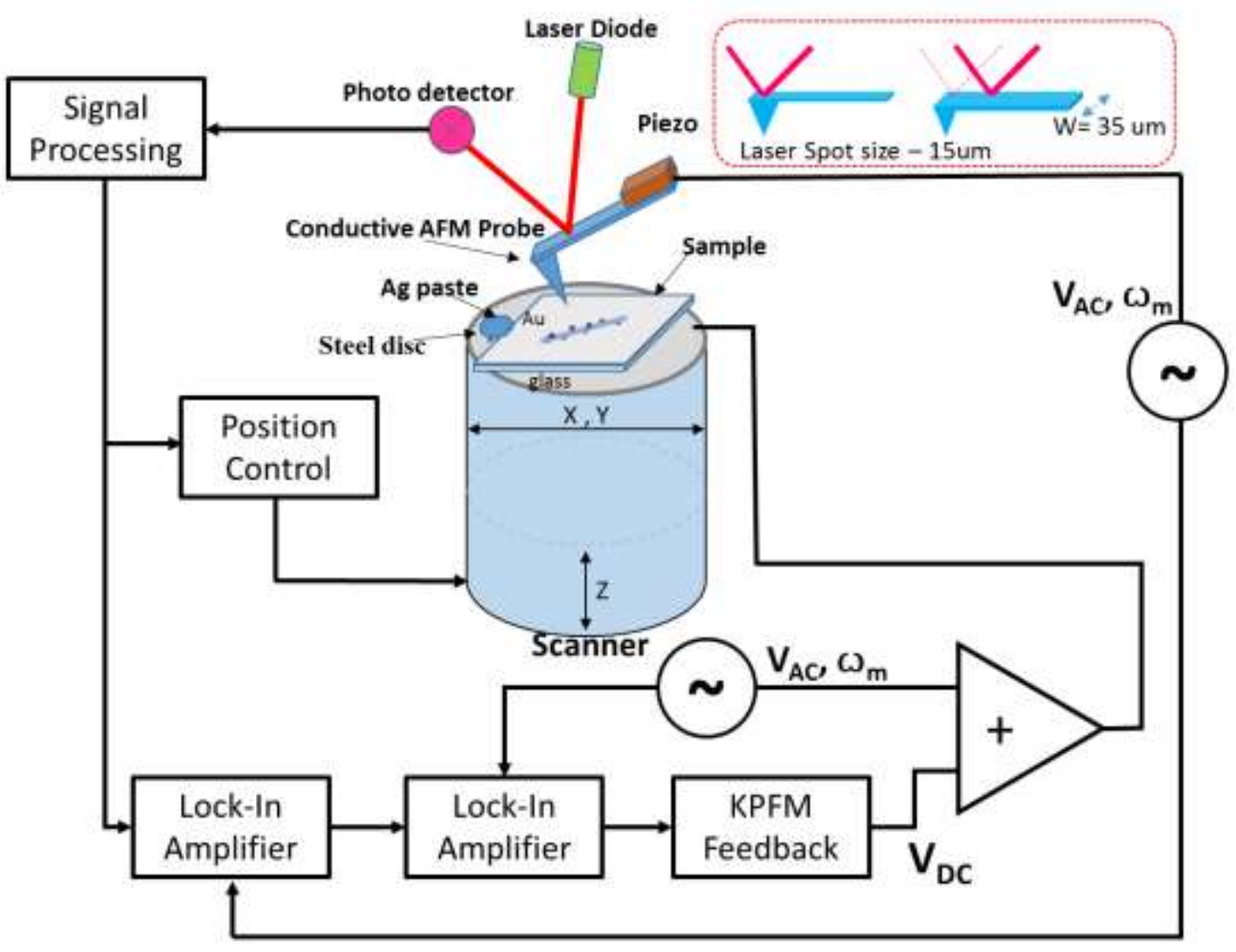

Figure S20. Schematic diagram for the PF-KPFM measurement setup.

PF-KPFM is a combination of PeakForce Tapping mode atomic force microscopy (AFM) and frequency modulated-Kelvin probe force microscopy (FM-KPFM). The schematic of a KPFM setup, used for the surface potential measurement, is shown in Figure S20. KPFM is based on the concept of a macroscale Kelvin Probe. This is a technique used to determine the surface potential of a material by exploiting the fact that when two materials with different work functions are brought into contact, their Fermi energies equalize via a flow of electrons from the one with a lower work function to the one with a higher work function. In the PF-KPFM set-up, a conductive AFM probe tip is used to form a capacitor 
with the sample surface. The potential established between tip and sample is then called the contact potential difference (CPD), or surface potential, and is equal to the work function difference between tip and sample. An external potential (called the backing potential) is applied to the tip or sample by the feedback loop until the surface charges disappear and the external potential become equal to the surface potential or CPD.

This can be easily demonstrated mathematically. When a conductive tip and a conductive sample form a capacitor, the electrostatic force between them can be expressed as:

$F=-\frac{1}{2} \frac{\partial C}{\partial z}(\Delta V)^{2}$

Where $F$ is the electric force, and $\Delta V$ is the potential difference between the conductive tip and the sample. If an external DC voltage $\left(V_{d c}\right)$ and AC voltage $V_{a c} \sin \left(\omega_{m} t\right)$ are applied between tip and sample, then the $\Delta V$ can be expressed as:

$\Delta V=V_{d c}-V_{c p d}+V_{a c} \sin \left(\omega_{m} t\right)$

Here, $V_{c p d}$ is the contact potential difference (surface potential) between tip and sample. Combining the above two equations the $F$ can be expressed as:

$F=\frac{\partial C}{\partial z}\left(\left(V_{d c}-V_{c p d}\right)^{2}+\frac{1}{2} V_{a c}{ }^{2}\right)+\frac{\partial C}{\partial z}\left(V_{d c}-V_{c p d}\right) V_{a c} \sin \left(\omega_{m} t\right)+\frac{1}{4} \frac{\partial C}{\partial z} V_{a c}{ }^{2} \cos \left(2 \omega_{m} t\right)$

When conductive cantilever is placed in an electric field, its effective spring constant is the sum of its natural spring constant $k$ and the electric force gradient. The force gradient changes the effective spring constant of the cantilever and can be given as:

$k_{e f f}=k-\frac{\partial F}{\partial z}$ 
Since we know the resonant frequency $(\omega)$ of a cantilever depends on the spring constant $(k)$ as:

$\omega=\sqrt{\frac{k}{m}}$

The change in the resonant frequency by the electric force gradient is:

$\Delta \omega \approx-\frac{\omega}{2 k}\left(\frac{\partial F}{\partial z}\right)$

And,

$\frac{\partial F}{\partial z}=\frac{\partial^{2} C}{\partial z^{2}}\left(\left(V_{d c}-V_{c p d}\right)^{2}+\frac{1}{2} V_{a c}{ }^{2}\right)+\frac{\partial^{2} C}{\partial z^{2}}\left(V_{d c}-V_{c p d}\right) V_{a c} \sin \left(\omega_{m} t\right)+\frac{1}{4} \frac{\partial^{2} C}{\partial z^{2}} V_{a c}{ }^{2} \cos \left(2 \omega_{m} t\right)$

Thus, from equation (S7) and (S8), the electric force gradient, created by the AC bias, modulates the resonant frequency of the cantilever at the $\mathrm{AC}$ bias frequency, $\omega_{m}$, and at its second harmonic, $2 \omega_{m}$. If a cantilever is oscillating at its resonant frequency, $\omega$, and simultaneously applying an AC bias at frequency $\omega_{m}$, usually only $2 \mathrm{kHz}$, the modulation of the resonant frequency gives rise to two pairs of sidebands at $\omega \pm \omega_{m}$, and $\omega \pm 2 \omega_{m}$ (see Equation S8). Using the amplitude of the sideband at $\omega \pm \omega_{\mathrm{m}}$ as KPFM feedback and adjusting the DC bias until they disappear leads to the point of $V_{d c}=V_{c p d}$, and the subsequent quantitative measurement of surface potential. To achieve this, two cascaded lock-in amplifiers have been used (figure S20). The first amplifier locks at the resonant frequency $(\omega)$, the phase output of which is fed into the second lock-in, which locks at the AC bias frequency $\left(\omega_{m}\right)$. The amplitude output (or KPFM feedback) of the second lock-in is therefore, the sum of $\omega \pm \omega_{m}$ (see Figure S20). PeakForce KPFM has an advantage over other KPFM modes in avoiding possible artifacts in a KPFM signal caused by topography crosstalk. The 
topography artifacts can be easily observed as abrupt changes in potential signal if there is any interference in the KPFM measurement due to being at to low a lift height.
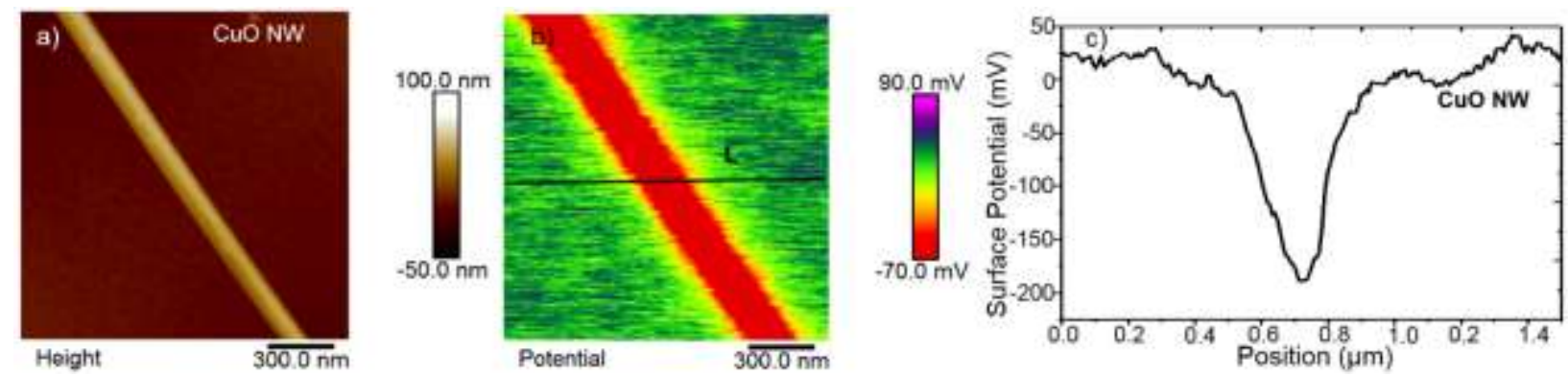

Figure S21. a) The height image of a $\mathrm{CuO} \mathrm{NW}$ on an $\mathrm{Au}$ substrate, b) potential image the corresponding nanowire shown in a) and, c) line profiles of the potential along the corresponding lines drawn in b).

a)
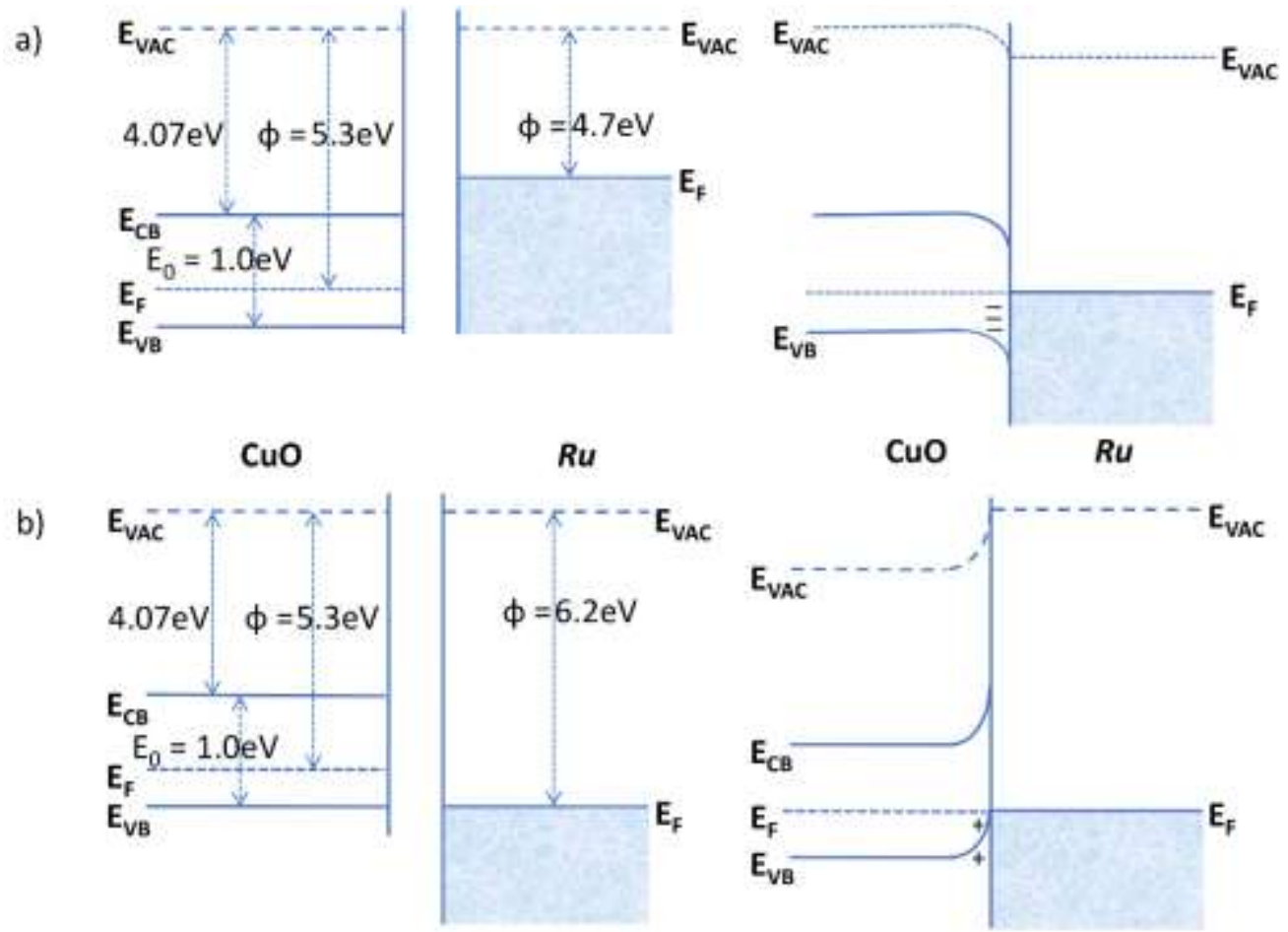

CuO

$\mathrm{RuO}_{2}$

Before Contact

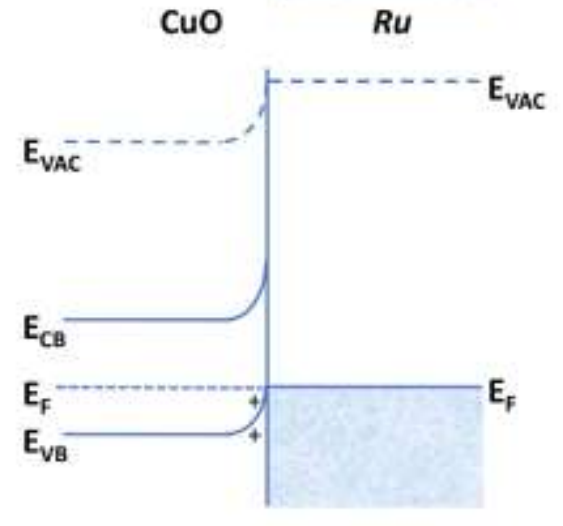

CuO

$\mathrm{RuO}_{2}$

After Contact

Figure S22. Band diagrams before and after contact between nanoclusters and nanowires for a) $\mathrm{Ru}$ and b) $\mathrm{RuO}_{2}$. 


\section{S8. Acetone gas sensing measurments}

a)

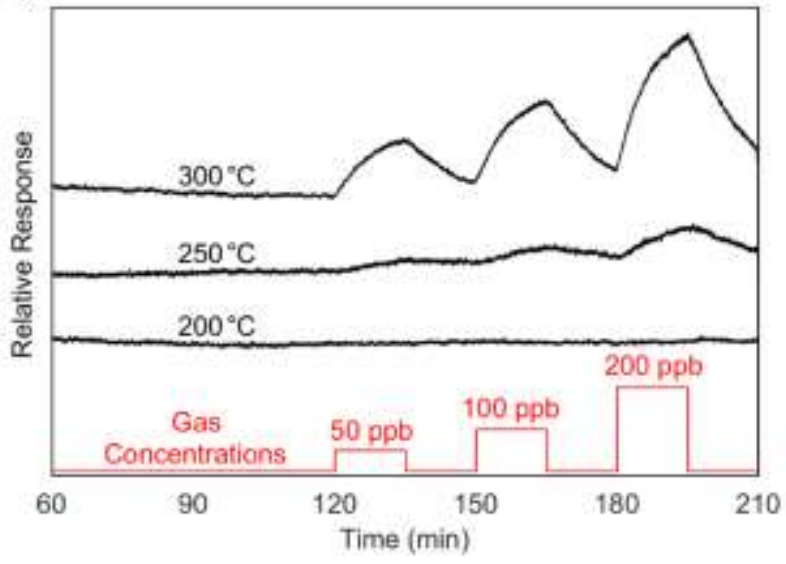

b)

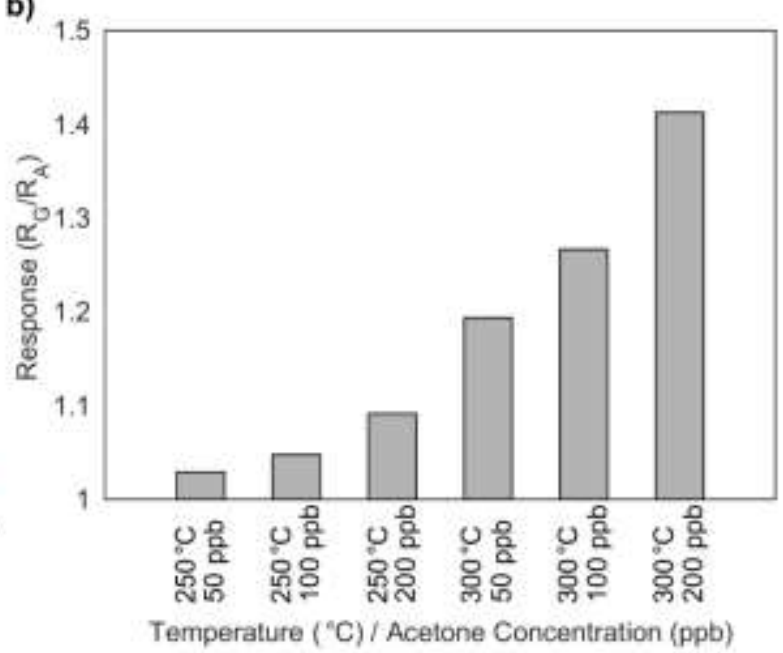

Figure S23. a) The relative resistance of a $\mathrm{CuO}$ NW device operated at various temperatures and multiple concentrations of acetone-dry air mixtures. b) Shows the response of the $\mathrm{CuO} N W$ device of acetone at temperatures of $250^{\circ} \mathrm{C}$ and $300^{\circ} \mathrm{C}$.
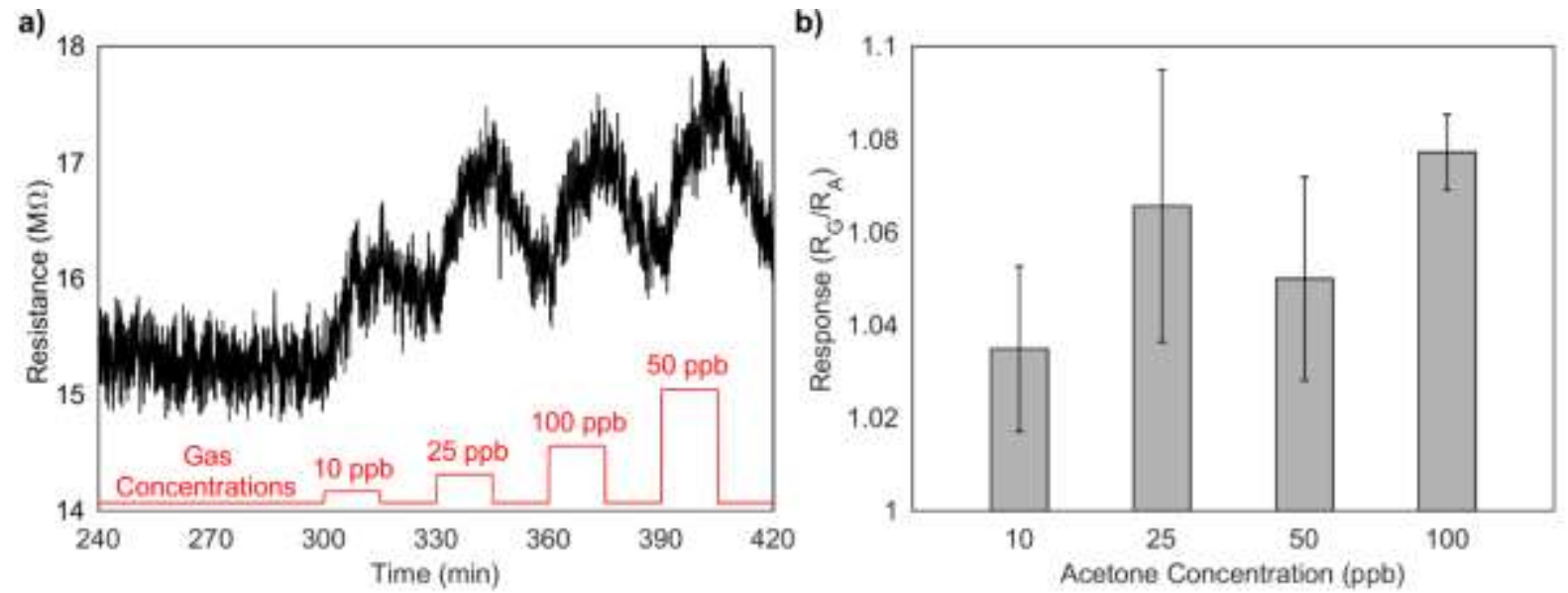

Figure S24. a) The resistance of an $\mathrm{Ru}$ decorated $\mathrm{CuO}$ NW device operated at a temperature of $200^{\circ} \mathrm{C}$ for various concentrations of acetone-dry air mixtures. b) Shows the average response of Ru-decorated $\mathrm{CuO} \mathrm{NW}$ devices (error bars indicate 1 standard deviation) for acetone gas. 


\section{S9. Band structure of $\mathrm{CuO}$ atomistic model}

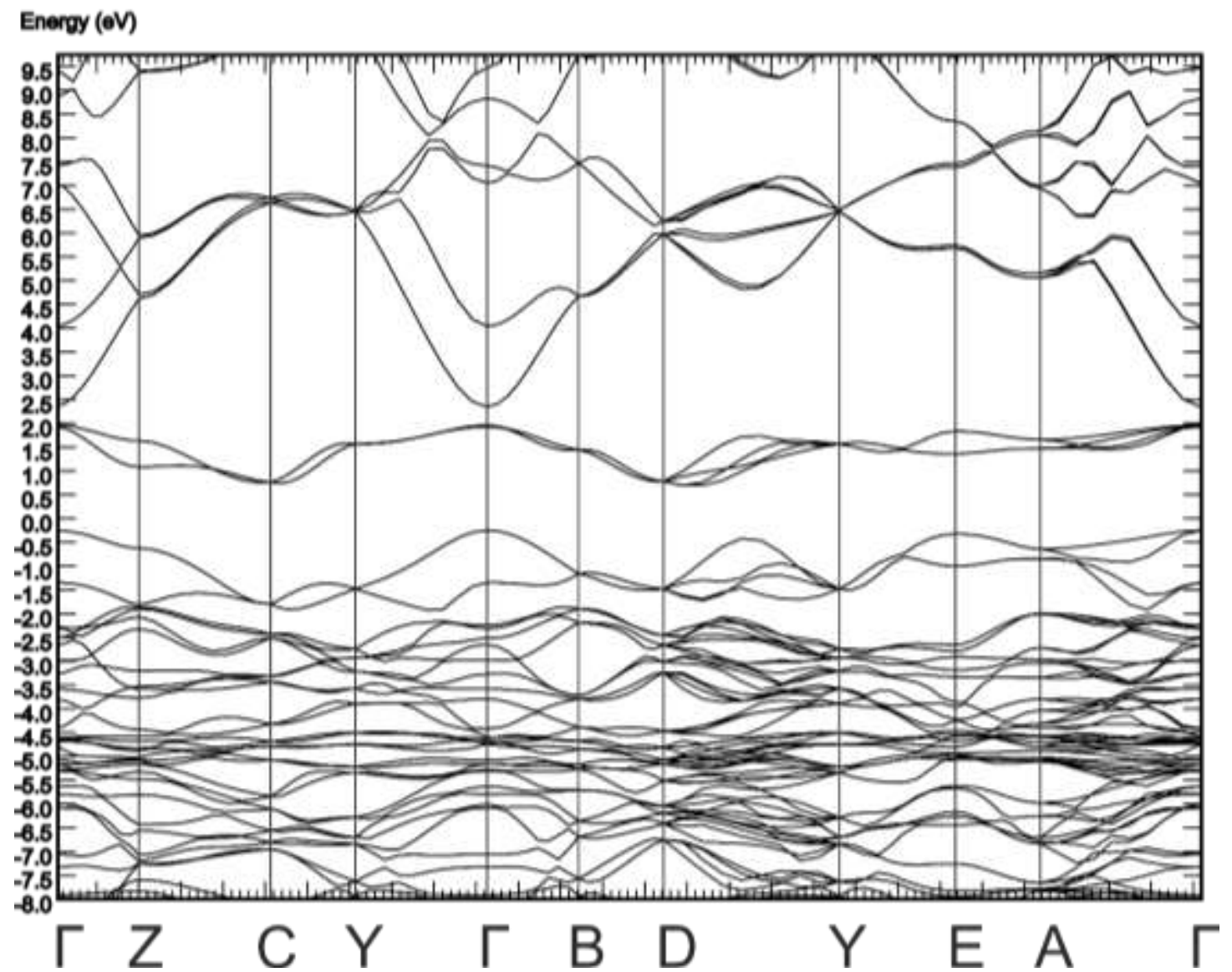

Figure S25. The calculated band structure of $\mathrm{CuO}$ along the high symmetry directions of the structure.

\section{S10. References}

1. Moulder, J. F.; Stickle, W. F.; Sobol, P. E.; Bomben, K. D., Handbook of X-Ray Photoelectron Epectroscopy: A Reference Book of Standard Spectra for Identification and Interpretation of XPS Data. ULVAC-PHI: Chigasaki, Japan, 1995.

2. Biesinger, M. C., Advanced Analysis of Copper X-Ray Photoelectron Spectra. Surf. Interface Anal. 2017, 49, 1325-1334.

3. Morgan, D. J., Resolving Ruthenium: XPS Studies of Common Ruthenium Materials. Surf. Interface Anal. 2015, 47, 1072-1079.

4. Ryden, W. D.; Lawson, A. W., Electrical Transport Properties of $\mathrm{IrO}_{2}$ and $\mathrm{Ru}_{2}$. Phys. Rev. $B$ 1970, 1, 1494-1500. 
5. Wertheim, G. K.; Guggenheim, H. J., Conduction-Electron Screening in Metallic Oxides: IrO 2 . Phys. Rev. B 1980, 22, 4680-4683.

6. $\quad$ Song, C.; Sakata, O.; Kumara, L. S. R.; Kohara, S.; Yang, A.; Kusada, K.; Kobayashi, H.; Kitagawa, H., Size Dependence of Structural Parameters in FCC and HCP Ru Nanoparticles, Revealed by Rietveld Refinement Analysis of High-Energy X-Ray Diffraction Data. Sci. Rep. 2016, 6, 31400

7. Nanba, Y.; Ishimoto, T.; Koyama, M., Structural Stability of Ruthenium Nanoparticles: A Density Functional Theory Study. J. Phys. Chem. C 2017, 121, 27445-27452.

8. Alyami, N. M.; LaGrow, A. P.; Anjum, D. H.; Guan, C.; Miao, X.; Sinatra, L.; Yuan, D.J.; Mohammed, O. F.; Huang, K.-W.; Bakr, O. M., Synthesis and Characterization of Branched FCC/HCP Ruthenium Nanostructures and Their Catalytic Activity in Ammonia Borane Hydrolysis. Cryst. Growth Des. 2018, 18, 1509-1516.

9. Stadelmann, P. A., EMS - A Software Package for Electron Diffraction Analysis and HREM Image Simulation in Materials Science. Ultramicroscopy 1987, 21, 131-145.

10. Pley, M.; Wickleder, M. S., Two Crystalline Modifications of $\mathrm{RuO}_{4}$. J. Solid State Chem. 2005, 178, 3206-3209. 